\title{
Cone decompositions of non-simple polytopes
}

\author{
José Agapito And Leonor Godinho
}

\begin{abstract}
A polytope cone decomposition expresses the characteristic function of a polytope as a sum of characteristic functions of convex cones associated to its faces. In this work we generalize the decompositions for simple polytopes introduced in [3] to any convex polytope.
\end{abstract}

\section{Introduction}

The interplay between simple convex lattice polytopes and projective toric varieties has been extensively studied. If a lattice polytope $P \subset \mathbb{R}^{d}$ is nonsimple (meaning that there is at least one vertex with more than $d$ incident edges), we can still associate to $P$ a projective toric variety, now with worse than orbifold singularities, by a generalization of the Delzant construction [6]. These singular toric varieties appear in many contexts. They can arise, for instance, as a result of singular symplectic reduction on toric manifolds (see for example [8] for the case of isolated singularities) and as Calabi Yau manifolds, with implications in mirror symmetry and string theory [11]. It is then interesting to extend the existing simple-polytope decompositions to non-simple polytopes.

Some of these formulas $[3,13]$ were obtained by exploring the relation between simple lattice regular polytopes and smooth toric varieties, and then generalized to all simple lattice polytopes using combinatorial methods. In particular, the decompositions obtained in [3] were suggested by the work of Witten on localization on critical values of the square of the moment map. This localization technique was developed by Paradan [16] and later generalized by Harada-Karshon [10].

Perhaps the most well-known polytope decomposition is the BrianchonGram formula [7] which expresses the characteristic function $\mathbf{1}_{P}$ of a convex polytope $P$ as the alternating sum of the characteristic functions of the 
tangent cones $\mathbf{C}_{F}$ at the faces of $P$; namely,

$$
\mathbf{1}_{P}=\sum_{F \subseteq P}(-1)^{\operatorname{dim} F} \mathbf{1}_{\mathbf{C}_{F}} .
$$

The Lawrence-Varchenko decomposition [14, 17], also known as polar decomposition, expresses the characteristic function of a convex simple polytope (only) in terms of the characteristic functions of polarized tangent cones at the vertices of $P$ (cones obtained by flipping some of the edge vectors emanating from each vertex, so that they all point in the same direction). In particular, we have

$$
\mathbf{1}_{P}=\sum_{\mathbf{v} \text { vertex of } P}(-1)^{m_{\mathbf{v}}} \mathbf{1}_{\mathbf{C}_{\mathbf{v}}^{\sharp}},
$$

where $m_{\mathbf{v}}$ is the number of edge vectors of $\mathbf{C}_{\mathbf{v}}$ which are flipped and $\mathbf{C}_{\mathbf{v}}^{\sharp}$ is the resulting flipped (or polarized) tangent cone at the vertex $\mathbf{v}$. This flipping process is determined by a direction specified by a choice of a special vector (called polarizing vector) that cannot be parallel to any of the affine lines generated by the edges of $P$. Formula (2) has been generalized by Haase [9] to non-simple polytopes.

Karshon, Sternberg and Weitsman [13] and Agapito [1] gave weighted versions of (2) (for simple polytopes), assigning weights to the faces of the polytope $P$ and of the polarized cones $\mathbf{C}_{\mathbf{v}}^{\sharp}$ in a consistent way (see Section 3 for a detailed explanation). A weighted version of (1) was also given in [2].

In [3] new polytope decompositions for simple polytopes were obtained that combine the above two formulas. As in (1), these decompositions express $\mathbf{1}_{P}$ in terms of characteristic functions of cones with the affine spaces generated by the faces of the polytope as apices. However, these cones are not polarized according to a unique direction. Indeed, a different vector is assigned to each face of $P$ and the generators of the corresponding tangent cone are flipped accordingly. These polarizing vectors are obtained by choosing a suitable starting point $\varepsilon$ (the same for all such vectors) and taking as end points the orthogonal projections $\beta\left(\varepsilon, \Delta_{F}\right)$ of $\varepsilon$ onto the affine spaces $\Delta_{F}$ generated by the different faces. The formulas thus obtained are

$$
\mathbf{1}_{P}^{w}=\sum_{F \subseteq P}(-1)^{m_{F}} \mathbf{1}_{P}\left(\beta\left(\varepsilon, \Delta_{F}\right)\right) \mathbf{1}_{\mathbf{C}_{F}^{\sharp}}^{w},
$$

where $m_{F}$ is the number of generators of $\mathbf{C}_{F}$ that are flipped, $\mathbf{1}_{\mathbf{C}_{F}^{\sharp}}^{w}$ is the weighted characteristic function of the polarized tangent cone $\mathbf{C}_{F}^{\sharp}$ at $F$, and 
$\mathbf{1}_{P}^{w}$ is the weighted characteristic function of the polytope $P$. In this work we generalize these decompositions to any convex polytope.

The idea is to argue as follows. If $V$ is a vector space of dimension $d$ and $P$ is a full-dimensional polytope in $V$, we write the tangent cone to $P$ at a non-simple face $F$ (meaning that $F$ is contained in a number of facets of $P$ greater than its codimension) as an intersection of simple cones with apex $\Delta_{F}$ determined by a regular triangulation $\mathcal{T}_{F}$ of the dual cone to $\mathbf{C}_{F}$ (also known as the normal cone at $F$ ). We then polarize these simple cones as described above and sum the weighted characteristic functions of the resulting polarized cones, taking into consideration the parity of the number of edges that are flipped, obtaining a function

$$
\mathbf{1}_{\mathcal{T}_{F}}^{w}:=\sum_{i}(-1)^{m_{F, i}} \mathbf{1}_{\mathbf{C}_{\sigma_{i}(F)}^{\sharp}}^{w} .
$$

The values of this function are independent of the choice of the regular triangulation $\mathcal{T}_{F}$ at all points which do not lie in any affine space generated by a non-simple face containing $F$ (cf. Proposition 6.6). Moreover, if we assign the same weight to all the hyperplanes generated by the facets of $P$, the values of $\mathbf{1}_{\mathcal{T}_{F}}^{w}$ are always independent of the triangulation, for all points in $V$. In all cases, denoting by $\mathbf{1}_{F}^{w}$ the restriction of $\mathbf{1}_{\mathcal{T}_{F}}^{w}$ to the set of points where this independence holds, and by $W$ the set of possible starting points of polarizing vectors (described in Section 6), we obtain the following result.

Theorem 1.1. Let $P \subset V$ be any convex full-dimensional polytope and let $S$ be the union of the affine spaces generated by the non-simple faces of $P$. Then, for $\varepsilon \in W$ and $\mathbf{x} \in V \backslash S$, we have

$$
\mathbf{1}_{P}^{w}(\mathbf{x})=\sum_{F \subseteq P} \mathbf{1}_{P}\left(\beta\left(\varepsilon, \Delta_{F}\right)\right) \mathbf{1}_{F}^{w}(\mathbf{x})
$$

where $\beta\left(\varepsilon, \Delta_{F}\right)$ is the orthogonal projection of $\varepsilon$ onto the affine space $\Delta_{F}$ generated by the face $F$. Moreover, when all the weights assigned to the hyperplanes generated by the facets of $P$ are equal to some fixed $q \in \mathbb{C}$, the value of the function on the RHS of (3) at $\mathbf{x} \in S$ is also independent of the choice of regular triangulation and is equal to

$$
\left\{\begin{array}{ll}
q^{\operatorname{codim} F_{\mathbf{x}}}-\left(r_{\mathbf{x}}-\operatorname{codim} F_{\mathbf{x}}\right) q^{\operatorname{codim} F_{\mathbf{x}}-1}(1-q) & \text { if } \mathbf{x} \in P \\
0 & \text { otherwise }
\end{array},\right.
$$


where $r_{\mathbf{x}}$ is the number of facets of $P$ that contain $\mathbf{x}$ and $F_{\mathbf{x}}$ is the face of $P$ such that $\mathbf{x} \in$ int $F_{\mathbf{x}}$. In particular, for $q=1$, the RHS of (3) is equal to $\mathbf{1}_{P}(\mathbf{x})$, the (unweighted) characteristic function of $P$, for every $\mathbf{x} \in V$.

This theorem is first proved for compatible choices of regular triangulations of the different inner normal cones to $P$ at its non-simple faces (cf. Proposition 6.2). Then, we use this fact to show that the functions $\mathbf{1}_{\mathcal{T}_{F}}^{w}$ are independent of triangulations, thus obtaining the final complete result as stated. The compatible choices of regular triangulations are obtained by considering a regular triangulation of the polar dual polytope $P^{*}$ [9], as explained in Section 6. Since the functions involved are independent of the triangulation, we do not have to worry about the compatibility of the regular triangulations used when applying Theorem 1.1. The proof of Proposition 6.2 is divided in two parts. Firstly, we show that the sum

$$
\sum_{F \subseteq P} \mathbf{1}_{P}\left(\beta\left(\varepsilon, \Delta_{F}\right)\right) \mathbf{1}_{\mathcal{T}_{F}}^{w}
$$

is independent of $\varepsilon$ (the starting point of all polarizing vectors), where the functions $\mathbf{1}_{\mathcal{T}_{F}}^{w}$ are constructed using compatible regular triangulations. This is done by a wall-crossing argument; i.e., by considering the effect of crossing a wall in the complement of the set of suitable values of $\varepsilon$. Secondly, we show that

$$
\mathbf{1}_{P}^{w}(\mathbf{x})=\sum_{F \subseteq P} \mathbf{1}_{P}\left(\beta\left(\varepsilon, \Delta_{F}\right)\right) \mathbf{1}_{\mathcal{T}_{F}}^{w}(\mathbf{x})
$$

holds for $\mathbf{x} \in V \backslash S$ by carefully picking a suitable value of $\varepsilon$ for each $\mathbf{x}$. In addition, since the sum in (4) is independent of $\varepsilon$ for every $\mathbf{x} \in V$, we compute this sum when $\mathbf{x} \in S$, in the particular case where the same weight is assigned to all the hyperplanes generated by the facets of $P$. A crucial step in this computation, as well as in the proof of (5) in the general situation, is Lemma 6.5, which states that, given a regular triangulation of the normal cone at a non-simple vertex, there exists a special simple polarized cone $\mathbf{C}_{\sigma_{i_{0}}(\mathbf{v})}^{\sharp}$ that contains all the other polarized cones $\mathbf{C}_{\sigma_{i}(\mathbf{v})}^{\sharp}$ determined by the triangulation.

Finally, let us point out that making a choice of compatible regular triangulations corresponds to performing simple deformations of the polytope $P$ by parallel translating some of its facets. This observation gives an alternative proof of Proposition 6.2 for $\mathrm{x} \in V \backslash S$. Nevertheless, if we want to extend this result to $V$, as we do when we assign the same weight to all the 
hyperplanes generated by the facets of $P$, it is crucial to use a wall-crossing argument (cf. Remark 6.9).

This paper is structured as follows. In Sections 3 and 4 we describe the polarizing process and the assignment of weights to the different faces of the polytope and of the polarized cones. Then, in Section 5, we describe the decompositions presented in [3] and, in Section 6, we generalize these formulas to any convex polytope.

\section{Preliminaries}

\subsection{Polytopes and convex cones}

Let $V$ be a real vector space of dimension $d$ with a lattice $\Lambda$. We refer to the elements of $\Lambda$ as integral points. An element $\mathbf{x} \in V$ is called rational if $k \mathrm{x} \in \Lambda$ for some integer $k \neq 0$. The space of rational elements of $V$ is denoted by $V_{\mathbb{Q}}$. A subspace $W \subset V$ is called rational if $W \cap \Lambda$ is a lattice in $W$, and an affine subspace of $V$ is called rational if it is a translate of a rational subspace by a rational element of $V$. The dual space $V^{*}$ is equipped with the dual lattice $\Lambda^{*}$ of $\Lambda$ :

$$
\Lambda^{*}:=\left\{\xi \in V^{*} \mid\langle\xi, \mathbf{x}\rangle \in \mathbb{Z} \text { for every } \mathbf{x} \in \Lambda\right\}
$$

If $S$ is a subset of $V$, then we denote by $\Delta_{S}$ the affine subspace generated by $S$. If $S$ consists of rational elements of $V$, then $\Delta_{S}$ is rational. Note that $\Delta_{S}$ may contain no integral points. We denote by $\operatorname{lin}(S)$ the vector subspace of $V$ parallel to $\Delta_{S}$ and by $S^{\perp}$ the subspace of $V^{*}$ orthogonal to $S$ :

$$
S^{\perp}=\left\{\xi \in V^{*} \mid\langle\xi, \mathbf{x}\rangle=0 \text {, for every } \mathbf{x} \in S\right\} .
$$

If $W$ is a subspace of $V$, the dual space $(V / W)^{*}$ is canonically identified with the subspace $W^{\perp} \subset V^{*}$. The vector space $V$ has a canonical Lebesgue measure $d \mathbf{x}$ for which the fundamental domain of $\Lambda$ has measure 1 . In particular, if $\left\{\mathbf{e}_{1}, \ldots, \mathbf{e}_{d}\right\}$ is a basis of $V$ such that

$$
\Lambda=\sum_{i=1}^{d} \mathbb{Z} \mathbf{e}_{i}
$$

then, for $\mathbf{x}=\sum_{i=1}^{d} x_{i} \mathbf{e}_{i} \in V$, we have $d \mathbf{x}=d x_{1} \cdots d x_{n}$.

A convex rational cone in $V$ is a closed convex cone $\sum_{i=1}^{k} \mathbb{R}_{+} \mathbf{w}_{i}$ which is generated by a finite number of elements $\mathbf{w}_{i} \in V_{\mathbb{Q}}$. An affine rational cone 
in $V$ is a translate of a convex rational cone in $V$ by a rational element of $V$. All cones considered in this work are convex and rational. We will simply refer to them as cones. A cone $\mathbf{C}$ (affine or not) is said to be pointed if it does not contain any straight line. We can then write

$$
\mathbf{C}=\left\{\mathbf{v}+\lambda_{1} \mathbf{w}_{1}+\cdots+\lambda_{k} \mathbf{w}_{k} \mid \lambda_{1}, \ldots, \lambda_{k} \geq 0\right\},
$$

where $\mathbf{v} \in V_{\mathbb{Q}}$ and $\mathbf{w}_{1}, \ldots, \mathbf{w}_{k} \in V_{\mathbb{Q}}$ generate the extreme rays of $\mathbf{C}$. These vectors $\mathbf{w}_{i}$, only defined up to a positive scalar, are called the generators of C. If a cone $\mathbf{C}$ is not pointed, then it contains an affine space of positive dimension. The maximal affine space contained in $\mathbf{C}$ is called the apex of C. The dimension of a cone $\mathbf{C}$ is the dimension of the affine space $\Delta_{\mathbf{C}}$ generated by $\mathbf{C}$. If $\operatorname{dim} \mathbf{C}=d$ then $\mathbf{C}$ is called a full-dimensional (or solid) cone, in which case $\Delta_{\mathbf{C}}=V$. Note that any affine rational cone $\mathbf{C}$ is the intersection of a finite number of half-spaces whose boundaries are affine rational hyperplanes meeting in (at least) one point.

A (rational) convex polytope $P \subset V$ is a bounded intersection of a minimal finite collection $\mathcal{B}(P)$ of closed half-spaces in $V$ (with rational boundaries). Throughout this paper we will assume that any polytope is rational, convex and full-dimensional. Let $\mathcal{F}$ be the set of closed faces of $P$. Then

$$
\mathcal{F}=\bigcup_{k=0}^{n} \mathcal{F}(k),
$$

where $\mathcal{F}(k)$ is the set of faces of $P$ of dimension $k$. Note that $\mathcal{F}(d)=\{P\}$ and that $\mathcal{F}(0)$ is the set of vertices of $P$. A face of codimension- 1 is called a facet. If $F$ is a face of $P$, we define the set

$$
\mathcal{F}^{F}:=\{\widetilde{F} \in \mathcal{F}(d-1) \mid F \subset \widetilde{F}\}
$$

as the set of facets of $P$ that contain $F$. For each facet $F \in \mathcal{F}(d-1)$, we write the affine hyperplane $\Delta_{F}$ as

$$
\Delta_{F}=\left\{\mathbf{x} \in V \mid\left\langle\eta_{F}, \mathbf{x}\right\rangle+\lambda_{F}=0\right\},
$$

where $\lambda_{F} \in \mathbb{R}$ and $\eta_{F} \in V^{*}$ is a normal vector to the facet $F$ such that

$$
P \subset\left\{\mathbf{x} \in V \mid\left\langle\eta_{F}, \mathbf{x}\right\rangle+\lambda_{F} \geq 0\right\}
$$

(i.e. $\eta_{F}$ is an inward-pointing normal vector). Note that $\eta_{F}$ is only determined up to multiplication by an element of $\mathbb{R}_{+}$. 
If $F$ is a face of $P$, we define the tangent cone to $P$ at $F$ as

$$
\mathbf{C}_{F}:=\left\{\mathbf{y}+r(\mathbf{x}-\mathbf{y}) \mid r \geq 0, \mathbf{y} \in \Delta_{F}, \mathbf{x} \in P\right\} \subset V .
$$

It is the smallest (full-dimensional) affine cone with apex $\Delta_{F}$ that contains $P$. Let the barrier cone of $F$ be the translate of $\mathbf{C}_{F}$ that contains $\operatorname{lin}(F)$. Then we define the dual cone $\mathbf{N}_{F}$ to $\mathbf{C}_{F}$ (also known as the normal cone at $F)$ as

$$
\mathbf{N}_{F}:=\left\{\xi \in V^{*} \mid\langle\xi, \mathbf{x}\rangle \geq 0 \text { for all } \mathbf{x} \text { in the barrier cone of } F\right\}
$$

Then

$$
\mathbf{N}_{F}=\sum_{\widetilde{F} \in \mathcal{F}^{F}} \mathbb{R}_{\geq 0} \eta_{\widetilde{F}} .
$$

Finally, considering the projection $\pi_{F}: V \rightarrow V / \operatorname{lin}(F)$, we define the transverse cone $\mathbf{T}_{F}$ of $P$ along $F$ as the cone in $V / \operatorname{lin}(F)$ given by $\pi_{F}\left(\mathbf{C}_{F}\right)$. It is a solid pointed affine cone in the quotient space $V / \operatorname{lin}(F)$ with vertex $\pi_{F}\left(\Delta_{F}\right)$. Note that if $F$ is a vertex of $P$, then the transverse cone $\mathbf{T}_{F}$ coincides with the tangent cone $\mathbf{C}_{F}$.

Let $\mathbf{C}$ be a solid cone in $V$. Given a face $F$ of $\mathbf{C}$, let $r_{F}^{\mathbf{C}}$ be the number of facets of $\mathbf{C}$ that contain $F$. A face $F$ of $\mathbf{C}$ will be called simple in $\mathbf{C}$ if

$$
r_{F}^{\mathbf{C}}=\operatorname{codim} F=d-\operatorname{dim} F
$$

The cone $\mathbf{C}$ is said to be a simple cone if all its faces are simple. Similarly, a polytope is called simple if the tangent cones to $P$ at its faces are all simple.

Given a full-dimensional polytope $P \subset V$, consider the (pointed) cone $\mathbf{C}(P)$ spanned by $P$; that is, the set of all real, non-negative linear combinations of elements of $P \times\{1\}$. The dual cone $\mathbf{C}(P)^{\vee}$ of $\mathbf{C}(P)$ is the cone whose extremal rays are generated by the outer normal vectors at the facets of $\mathbf{C}(P)$. If $P$ contains the origin, $\mathbf{C}(P)^{\vee}$ is itself spanned by a polytope, namely

$$
P^{\vee}=\left\{\xi \in V^{*} \mid\langle\xi, \mathbf{x}\rangle \leq 1 \text { for all } \mathbf{x} \in P\right\},
$$

called the polar of $P$ (see for instance [12] for details). For an arbitrary polytope $P$, there is a translate $Q$ such that the origin belongs to the relative interior of $Q$ relative to its affine hull $A$. It is then possible to take the polar $Q^{\vee}$ of $Q$ in $A$. Any polytope $Q^{\vee}$ formed in this way is usually denoted by $P^{*}$ and referred to as the polar dual of $P$. There are many different choices for $P^{*}$, but they are all combinatorially equivalent. Note that in this 
construction a $k$-face of $P$ corresponds to a $(d-(k+1))$-face of $P^{*}$ and vice-versa (cf. Figure 1).

$\begin{array}{ccc}\begin{array}{c}\text { Facets } \\ \text { of } \boldsymbol{P}\end{array} & \begin{array}{c}\text { Normal } \\ \text { vectors }\end{array} & \begin{array}{c}\text { Vertices } \\ \text { of } \boldsymbol{P}^{*}\end{array} \\ \text { aeb } & \eta_{1} & 1 \\ \text { bec } & \eta_{2} & 2 \\ \text { ced } & \eta_{3} & 3 \\ \text { dea } & \eta_{4} & 4 \\ \text { bfa } & \eta_{5} & 5 \\ \text { cfb } & \eta_{6} & 6 \\ \text { dfc } & \eta_{7} & 7 \\ \text { afd } & \eta_{8} & 8\end{array}$

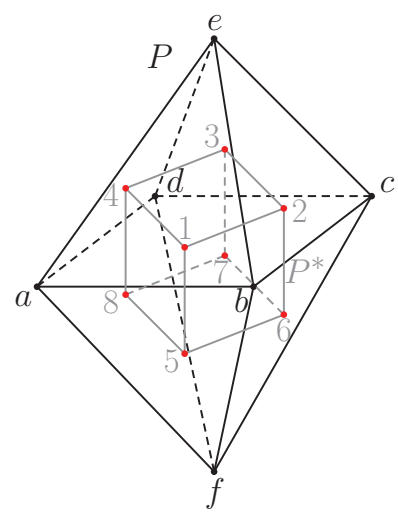

Figure 1: Polar duality for the octahedron and the cube.

\section{Polarization of simple tangent cones}

We will, from now on, fix a scalar product on $V$ and consider the associated isomorphism $j: V^{*} \rightarrow V$. Given a solid convex rational polytope $P \subset V$ let $W \subset V \backslash \partial P$ be the set of points $\varepsilon \in V \backslash \partial P$ such that, for every face $F$ of $P$, the $j$-orthogonal projection $\beta\left(\varepsilon, \Delta_{F}\right)$ of $\varepsilon$ onto $\Delta_{F}$ (defined by the scalar product) is not in any affine space generated by a proper subface of $F$. Then, fixing $\varepsilon \in W$ and considering a simple face $F$ of $P$ (i.e. one with a simple tangent cone), the vector

$$
\beta_{F}:=\beta\left(\varepsilon, \Delta_{F}\right)-\varepsilon \in V
$$

is the direction vector used to polarize the tangent cone $\mathbf{C}_{F}$ (see examples in Figure 2). To be more explicit, we start by taking the generators $\alpha_{F, j}$ of the transverse (pointed) cone $\mathbf{T}_{F} \subset V / \operatorname{lin}(F)$ and then we consider the vectors

$$
\alpha_{F, j}^{+}:=\epsilon_{F, j} \alpha_{F, j} \in V / \operatorname{lin}(F)
$$

such that $\left\langle j^{-1}\left(\pi_{F}\left(\beta_{F}\right)\right), \alpha_{F, j}^{+}\right\rangle>0$, where $\epsilon_{F, j} \in\{ \pm 1\}$. We then define the polarized tangent cone $\mathbf{C}_{F}^{\sharp}$ to $P$ at $F$ as the affine cone in $V$ with apex $\Delta_{F}$ 
whose image by $\pi_{F}$ is

$$
\pi_{F}\left(\mathbf{C}_{F}^{\sharp}\right)=\pi_{F}\left(\Delta_{F}\right)+\sum_{j=1}^{r_{F}} \mathbb{R}_{\geq 0} \alpha_{F, j}^{+},
$$

where $r_{F}=\left|\mathcal{F}^{F}\right|=\operatorname{codim} F$.

Remark 3.1. Note that, by definition of $W,\left\langle\left(j^{-1} \circ \pi_{F}\right)\left(\beta_{F}\right), \alpha_{F, j}\right\rangle \neq 0$ for every $j=1, \ldots, r_{F}$. Indeed, if $\operatorname{codim} F \neq 0$ and $\alpha_{F, j} \in V / \operatorname{lin}(F)$ is a generator of $\mathbf{T}_{F}$, then $\left\langle\left(j^{-1} \circ \pi_{F}\right)\left(\beta_{F}\right), \alpha_{F, j}\right\rangle=0$ would imply that $j^{-1}\left(\beta_{F}\right) \in$ $\operatorname{lin}(\widetilde{F})^{\perp}$ for some other face $\widetilde{F}$ of $P$ with $\operatorname{dim} \widetilde{F}=\operatorname{dim} F+1$ and

$$
\pi_{F}\left(\Delta_{\widetilde{F}}\right)=\pi_{F}\left(\Delta_{F}\right)+\mathbb{R}_{\geq 0} \alpha_{F, j}
$$

Then, since $\beta\left(\varepsilon, \Delta_{F}\right) \in \Delta_{F}$, the point $\varepsilon$ would be in a hyperplane $j$ perpendicular to $\Delta_{\widetilde{F}}$, which is impossible by the definition of $W$ (the $j$ orthogonal projection of $\varepsilon$ onto $\Delta_{\widetilde{F}}$ would be in $\Delta_{F}$ and $F$ is a proper subface of $\widetilde{F})$.

\section{Weighted characteristic functions}

We will now see how to define weighted characteristic functions for $P$ and its polarized tangent simple cones (at simple faces). First, let us number the facets of $P$ as $F_{1}, \ldots, F_{N_{P}}$ and let $\Delta_{i}:=\Delta_{F_{i}}$ be the hyperplanes generated by the $F_{i}$. For each $i \in I:=\left\{1, \ldots, N_{P}\right\}$, one assigns a weight

$$
w_{i}(\mathbf{x}):=\left\{\begin{array}{ll}
q_{i} \in \mathbb{C} & \text { if } \mathbf{x} \in \Delta_{i} \\
1 & \text { if } \mathbf{x} \in V \backslash \Delta_{i}
\end{array},\right.
$$

where $q_{1}, \ldots, q_{N_{F}}$ are complex numbers chosen arbitrarily. Then, one defines the weighted characteristic function of $P$ associated to this choice of weights as

$$
\mathbf{1}_{P}^{w}(\mathbf{x}):= \begin{cases}\prod_{i=1}^{N_{P}} w_{i}(\mathbf{x}) & \text { if } \mathbf{x} \in P \\ 0 & \text { otherwise }\end{cases}
$$

For polarized tangent simple cones at simple faces of $P$ one proceeds as follows. Let $I_{F} \subset I$ be the index set of the collection of hyperplanes generated 
by the facets of $\mathbf{C}_{F}$

$$
I_{F}:=\left\{i \in I \mid F_{i} \in \mathcal{F}^{F}\right\}
$$

For each $i \in I_{F}$, let $H_{i}$ be the halfspace that contains $\mathbf{C}_{F}^{\sharp}$ and has boundary $\Delta_{i}$. Considering $H_{i}^{+}:=H_{i} \backslash \Delta_{i}$ one sets

(9) $\quad q_{i}^{\sharp}=\left\{\begin{array}{ll}q_{i} & \text { if } H_{i}^{+} \cap P \neq \emptyset \\ 1-q_{i} & \text { if } H_{i}^{+} \cap P=\emptyset\end{array} \quad\right.$ and $\quad w_{i}^{\sharp}(\mathbf{x})=\left\{\begin{array}{ll}q_{i}^{\sharp} & \text { if } \mathbf{x} \in \Delta_{i} \\ 1 & \text { if } \mathbf{x} \in V \backslash \Delta_{i}\end{array}\right.$.

One then defines the weighted characteristic function of the simple cone as

$$
\mathbf{1}_{\mathbf{C}_{F}^{w}}(\mathbf{x}):=\left\{\begin{array}{ll}
\prod_{i \in I_{F}} w_{i}^{\sharp}(\mathbf{x}), & \text { if } \mathbf{x} \in \mathbf{C}_{F}^{\sharp} \\
0, & \text { otherwise }
\end{array} .\right.
$$

(cf. [1, 3] for details and Figure 2 for examples). The usual characteristic functions can be obtained from the weighted ones by making all the $q_{i}$ equal to 1 , in which case one has $\mathbf{1}_{\mathbf{C}_{F}^{\sharp}}^{w}(\mathbf{x})=0$ whenever $\mathbf{x}$ is in a facet of $\mathbf{C}_{F}^{\sharp}$ lying in the boundary of one of the half spaces $H_{i}^{+}$with $H_{i}^{+} \cap P=\varnothing$.

\section{Decomposition formulas}

We now present the polytope decompositions introduced in [3] for simple polytopes. For each $\varepsilon \in W$, the direction vectors $\beta_{F}$ defined in (7) are used to polarize the tangent cones at the corresponding faces of $P$. Note that the decomposition formula in (11) only takes into account the polarized tangent cones at faces $F$ for which the $j$-orthogonal projection of $\varepsilon$ onto $F$ is in $P$.

Theorem 5.1. Let $P \subset V$ be a convex full-dimensional simple polytope and let $\varepsilon \in W$. Then,

$$
\mathbf{1}_{P}^{w}=\sum_{F \subseteq P}(-1)^{m_{F}} \mathbf{1}_{P}\left(\beta\left(\varepsilon, \Delta_{F}\right)\right) \mathbf{1}_{\mathbf{C}_{F}^{\sharp}}^{w},
$$

where the sum is taken over the faces $F$ of $P$, where $\Delta_{F}$ is the affine space generated by the face $F$, where $\mathbf{C}_{F}^{\sharp}$ is the polarized tangent cone at $F$ with respect to the direction vector $\beta_{F}=\beta\left(\varepsilon, \Delta_{F}\right)-\varepsilon$ (with $\beta\left(\varepsilon, \Delta_{F}\right.$ ) being the orthogonal projection of $\varepsilon$ onto $\Delta_{F}$ ), and where $m_{F}$ is the number of generators of the transverse cone $\mathbf{T}_{F}$ that are flipped by polarization. 


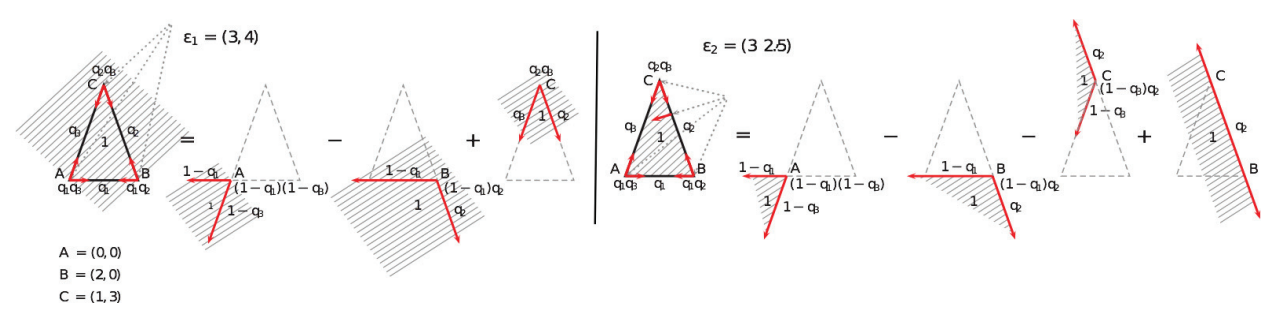

Figure 2: Polytope decompositions for two different values of $\varepsilon$.

If the tangent cones to $P$ at the different faces $F$ are polarized using the vectors $-\beta_{F}$ instead of $\beta_{F}$, one obtains the following polytope decompositions:

$$
\mathbf{1}_{P}^{w}=\sum_{F \subseteq P}(-1)^{m_{F}+\operatorname{dim} F} \mathbf{1}_{P}\left(\beta\left(\varepsilon, \Delta_{F}\right)\right) \mathbf{1}_{\mathbf{C}_{F}^{\sharp}}^{w} .
$$

We show in [3] that a region $R \subset W$ can be chosen such that, if $\varepsilon \in R$, the resulting decomposition in (12) becomes the Lawrence-Varchenko formula. Moreover, when

$$
A:=\operatorname{int}\left(\left(\bigcap_{\mathbf{v}}\left(\mathbf{v}+j^{-1}\left(\mathbf{N}_{\mathbf{v}}\right)\right)\right) \cap P\right) \neq \varnothing,
$$

where, for a given vertex $\mathbf{v}, \mathbf{N}_{\mathbf{v}}$ is the dual cone to $\mathbf{C}_{\mathbf{v}}$, we can take $\varepsilon \in A$ and obtain the Brianchon-Gram formula (1) from (12).

\section{Non-simple polytopes}

In this section we generalize the polytope decompositions presented in the previous section to any convex polytope $P$. Recall that a face $F$ of $P$ is simple when the number of facets of $P$ containing $F$ equals the codimension of $F$. We will call a face $F$ of $P$ non-simple when the number of facets of $P$ containing $F$ is greater than the codimension of $F$.

Let us label the facets of $P$ as $F_{1}, \ldots, F_{N_{P}}$, denote by $\eta_{i} \in V^{*}$ the normalized inner normal vectors $\eta_{F_{i}}$ to $F_{i}$, and write $\lambda_{i}:=\lambda_{F_{i}}$ (here $\eta_{F_{i}}$ and $\lambda_{F_{i}}$ are as in (6)). Then $P$ is the intersection of $N_{P}$ half-spaces

$$
P=\bigcap_{1 \leq i \leq N_{P}}\left\{\mathbf{x} \in V \mid\left\langle\eta_{i}, \mathbf{x}\right\rangle+\lambda_{i} \geq 0\right\} .
$$


Let $F$ be a non-simple face of $P$ and consider the corresponding set of facets $\mathcal{F}^{F}$. The cone $\mathbf{N}_{F}$, generated by the normal vectors $\eta_{i}$ to $F_{i} \in \mathcal{F}^{F}$, is the dual cone to $\mathbf{C}_{F}$. Let us choose a regular triangulation $\mathcal{T}_{F}$ of this cone; i.e., a face-to-face subdivision of $\mathbf{N}_{F}$ into simplicial cones $\sigma_{1}(F), \ldots, \sigma_{L_{F}}(F)$ for which there exists a convex piecewise linear function on $\mathbf{N}_{F}$ with the $\sigma_{i}(F) \mathrm{s}$ as domains of linearity (cf. $[5,15]$ for details). Note that $L_{F}=r_{F}-$ $(\operatorname{codim} F-1)$, where $r_{F}:=\left|\mathcal{F}^{F}\right|=r_{F}^{\mathbf{C}_{F}}$.

The tangent cone $\mathbf{C}_{F}$ can be written as the intersection of the simple cones $\mathbf{C}_{\sigma_{i}(F)}$ with apex $\Delta_{F}$,

$$
\mathbf{C}_{F}=\bigcap_{i=1}^{L_{F}} \mathbf{C}_{\sigma_{i}(F)}
$$

where

$$
\mathbf{C}_{\sigma_{i}(F)}:=\left\{\mathbf{x} \in V \mid\left\langle\eta_{j}, \mathbf{x}\right\rangle+\lambda_{j} \geq 0, j \in I_{F, i}\right\}
$$

with

$$
I_{F, i}:=\left\{j \in\left\{1, \ldots, N_{P}\right\} \mid F_{j} \in \mathcal{F}^{F} \text { and } \eta_{j} \in \sigma_{i}(F)\right\}
$$

(cf. Figure 3 for an example with $\operatorname{dim} F=0$ ).

Triangulation

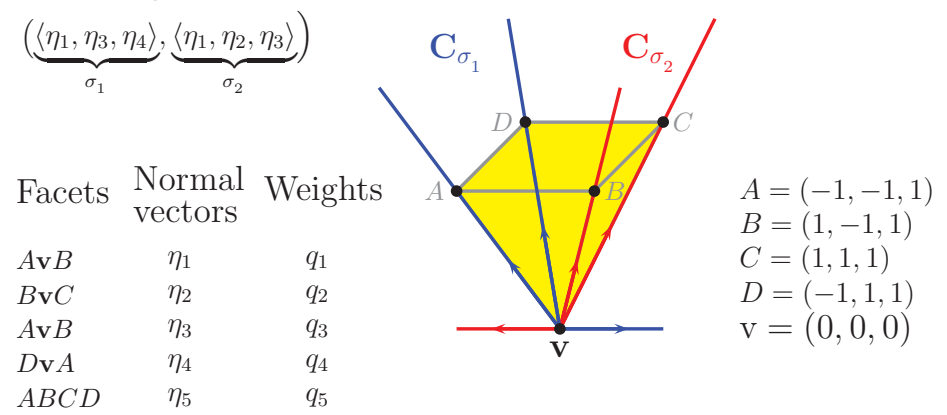

Figure 3: Example of a triangulation and the associated cones $\mathbf{C}_{\sigma_{i}}(\mathbf{v})$.

Choosing $\varepsilon \in V \backslash \partial P$ so that the $j$-orthogonal projection $\beta\left(\varepsilon, \Delta_{F}\right)$ of $\varepsilon$ onto $\Delta_{F}$ is not in any affine space generated by a proper subface of $F$, we can polarize the simple cones $\mathbf{C}_{\sigma_{i}(F)}$ as in Section 3. Let $m_{F, i}$ be the number of edges of the transverse cone of $\mathbf{C}_{\sigma_{i}(F)}$ at $F$ that are flipped in the polarizing process. Then, for each $j \in I_{F, i}$, we consider the half-space $H_{j}$ that contains $\mathbf{C}_{\sigma_{i}(F)}^{\sharp}$ and has boundary $\Delta_{j}:=\Delta_{F_{j}}$. We set $H_{j}^{\sharp}:=H_{j} \backslash \Delta_{j}$ and define $q_{j}^{\sharp}$ 
and $w_{j}^{\sharp}$ as in $(9)$. The weighted characteristic function of the simple cone $\mathbf{C}_{\sigma_{i}(F)}^{\sharp}$ is then

$$
\mathbf{1}_{\mathbf{C}_{\sigma_{i}(F)}^{\sharp}}^{w}(\mathbf{x}):=\left\{\begin{array}{ll}
\prod_{j \in I_{F, i}} w_{j}^{\sharp}(\mathbf{x}) & \text { if } \mathbf{x} \in \mathbf{C}_{\sigma_{i}(F)}^{\sharp} \\
0 & \text { otherwise }
\end{array} .\right.
$$

Finally we define the functions

$$
\mathbf{1}_{\mathcal{T}_{F}}^{w}:=\sum_{i=1}^{L_{F}}(-1)^{m_{F, i}} \mathbf{1}_{\mathbf{C}_{\sigma_{i}(F)}^{\sharp}}^{w} .
$$

Remark 6.1. For a simple face $F$ the tangent cone $\mathbf{C}_{F}$ is already a simple cone. Hence $\mathbf{C}_{F}=\mathbf{C}_{\sigma_{1}(F)}$, which allows us to write (15) as

$$
\mathbf{1}_{\mathcal{T}_{F}}^{w}=(-1)^{m_{F, 1}} \mathbf{1}_{\mathbf{C}_{\sigma_{1}(F)}^{\sharp}}^{w}=(-1)^{m_{F}} \mathbf{1}_{\mathbf{C}_{F}^{\sharp}}^{w} .
$$

Denoting by $S_{F}$ the union of the affine spaces generated by non-simple faces of $P$ that contain $F$, we will see later that the values of the function $\mathbf{1}_{\mathcal{T}_{F}}^{w}$ on $V \backslash S_{F}$ do not depend on the triangulation $\mathcal{T}_{F}$. For this reason, we will simply denote the function $\mathbf{1}_{\mathcal{T}_{F}}^{w}$ by $\mathbf{1}_{F}^{w}$.

However, before proving the last assertion, we have to show that our decompositions hold for non-simple polytopes using compatible choices of regular triangulations for the different dual cones to the tangent cones at non-simple faces of $P$. To do this, we proceed as follows. First, we take $P^{*}$, a polar dual polytope of $P$, and consider a regular triangulation $\mathcal{T}$ of $P^{*}$ (cf. Figure 4). Given a non-simple face $F \in \mathcal{F}(k)$, consider the corresponding $(d-(k+1))$-face of $P^{*}$, here denoted by $F^{*}$. The restriction of the triangulation $\mathcal{T}$ to $F^{*}$ decomposes this face into $(d-(k+1))$-simplices (each with $d-k$ vertices). Numbering the vertices of $P^{*}$ as $v_{1}, \ldots, v_{N_{P}}$ and the corresponding facets of $P$ by $F_{1}, \ldots, F_{N_{P}}$, let $I_{F^{*}} \subset\left\{1, \ldots, N_{P}\right\}$ be the index set of the vertices of $F^{*}$ (note that $\left|I_{F^{*}}\right|=r_{F}$ ). Then $\mathcal{T}$ defines a subdivision of $I_{F^{*}}$ into sets $I_{F, 1}, \ldots, I_{F, L_{F}}$ with $L_{F}:=r_{F}-\operatorname{codim} F+1$. Since the vertices of $P^{*}$ correspond to facets of $P$ and, in particular, the vertices of $F^{*}$ correspond to the facets of $P$ that contain $F$, this subdivision, in turn, gives us a regular triangulation $\mathcal{T}_{F}$ of $\mathbf{N}_{F}$, the dual cone to $\mathbf{C}_{F}$. Indeed, the triangulation of $\mathbf{N}_{F}$ is given by

$$
\mathcal{T}_{F}=\left\{\sigma_{1}(F), \ldots, \sigma_{L_{F}}(F)\right\}
$$


where each $\sigma_{i}(F)$ is the simple cone generated by the normal vectors $\eta_{j}$ to the facets $F_{j}$ of $P$ with $j \in I_{F, i}$ (all of which contain $F$ ).

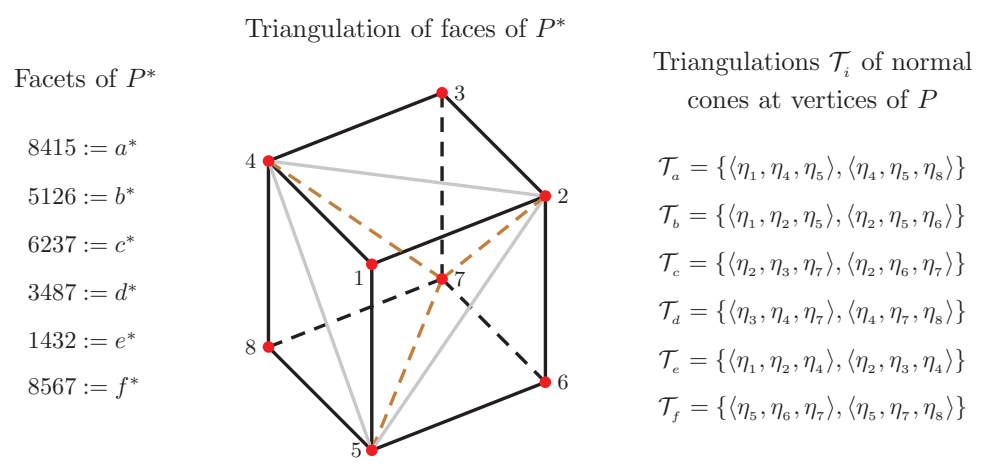

Figure 4: A regular triangulation of the dual polytope of the octahedron.

Finally, before stating and proving our result for these particular choices of triangulations, one has to establish the region $W \subset V$ where the initial point $\varepsilon$ of the polarizing vectors for the different tangent cones can be chosen from. For that, consider the set of affine spaces

$$
B:=\left\{\bigcap_{i \in I} \Delta_{i} \mid I \subset\left\{1, \ldots, N_{P}\right\}\right\}
$$

formed by all possible intersections of hyperplanes generated by facets of $P$. To ensure that the polarizing vectors $\left(j^{-1} \circ \pi_{F}\right)\left(\beta_{F}\right)$, with $\beta_{F}:=\beta\left(\varepsilon, \Delta_{F}\right)-$ $\varepsilon$, are not $j$-perpendicular to any generator of the transverse cone of $\mathbf{C}_{\sigma_{i}(F)}^{\sharp}$ at $F$, one takes $\varepsilon \in W$, where $W$ is now the set of points $\varepsilon$ in $V \backslash \partial P$ such that, for each $\Delta \in B$, the $j$-orthogonal projection $\beta(\varepsilon, \Delta)$ of $\varepsilon$ onto $\Delta$ is not in any proper affine subspace of $\Delta$ contained in $B$.

Using the functions defined in (10) and (15), a regular triangulation $\mathcal{T}$ of the polar dual polytope $P^{*}$, and the region $W$ defined above, one obtains the following proposition.

Proposition 6.2. Let $P \subset V$ be any convex polytope and let $S$ be the union of the affine spaces generated by non-simple faces of $P$. Moreover, let $\mathcal{T}$ be a regular triangulation of the polar dual polytope $P^{*}$, and consider its restrictions to regular triangulations $\mathcal{T}_{F}$ of the dual cones to the tangent 
cones of $P$ at non-simple faces $F$. Then, on $V \backslash S$ and for $\varepsilon \in W$, we have

$$
\mathbf{1}_{P}^{w}=\sum_{F \subseteq P} \mathbf{1}_{P}\left(\beta\left(\varepsilon, \Delta_{F}\right)\right) \mathbf{1}_{\mathcal{T}_{F}}^{w} \text { on } V \backslash S,
$$

where the sum is taken over the faces $F$ of $P$.

Moreover, in the particular case where all the weights $q_{i}\left(i=1, \ldots, N_{P}\right)$ are equal to some fixed value $q \in \mathbb{C}$, the sum on the $R H S$ of (16), at $\mathbf{x} \in S$, is equal to

$$
\begin{cases}q^{\operatorname{codim} F_{\mathbf{x}}}-\left(r_{\mathbf{x}}-\operatorname{codim} F_{\mathbf{x}}\right) q^{\operatorname{codim} F_{\mathbf{x}}-1}(1-q) & \text { if } \mathbf{x} \in P \\ 0 & \text { otherwise }\end{cases}
$$

where $F_{\mathbf{x}}$ is the face of $P$ such that $\mathbf{x} \in \operatorname{int}\left(F_{\mathbf{x}}\right)$ and $r_{\mathbf{x}}=\left|\mathcal{F}^{F_{\mathbf{x}}}\right|$ is the number of facets of $P$ that contain $\mathbf{x}$.

In particular, if $q=1$, we obtain

$$
\mathbf{1}_{P}=\sum_{F \subseteq P} \mathbf{1}_{P}\left(\beta\left(\varepsilon, \Delta_{F}\right)\right) \mathbf{1}_{\mathcal{T}_{F}}
$$

for every point $\in V$, where $\mathbf{1}_{P}$ is the (unweighted) characteristic function of $P$.

Remark 6.3. Note that, if $P$ is a simple polytope, we get back the decompositions in Theorem 5.1 (cf. Remark 6.1).

Remark 6.4. As it will become clear in the proof below, the equality in (16) still holds for points $\mathbf{x} \in S \cap P^{c}$ (where we have both sides equal to zero). However, the values of the functions $\mathbf{1}_{\mathcal{T}_{F}}^{w}$ at these points will not, in general, be independent of the triangulation.

Proof. As in the proof of Theorem 5.1 presented in [3], we show this result in two steps. First we show that the RHS of (16) is independent of the choice of $\varepsilon$. Then, for each $\mathbf{x} \in V \backslash S$, we find an $\varepsilon$ for which (16) holds. We choose this order because, with the first step, we will simultaneously be proving that the sum on the RHS of the weighted Lawrence-Varchenko decomposition for non-simple polytopes is independent of the choice of polarizing vector (since these are particular cases of (16)), which will be needed in the proof of the second step. The fact that the Lawrence-Varchenko decomposition for non-simple polytopes is a particular case of (16) is explained in detail in Remark 6.7. 
Step 1: The complement $W^{c}$ of $W$ is a finite family of walls of codimension $1, W^{c}=E_{1} \cup \cdots \cup E_{K} \cup \mathcal{F}(d-1)$. Let $\varepsilon_{1}$ and $\varepsilon_{2}$ be in two contiguous regions and let $E$ be its common wall. Let $\varepsilon_{t}$ be any path in $V$ from $\varepsilon_{1}$ to $\varepsilon_{2}$ that crosses $E$ once and does not cross any other wall. Given a proper face $F$ of $P$ and a generator $\alpha_{F, k} \in V / \operatorname{lin}(F)$ of the transverse cone to one of the cones $\mathbf{C}_{\sigma_{i}(F)}$ defined by the corresponding triangulation $\mathcal{T}_{F}$, the sign of

$$
\left\langle\left(j^{-1} \circ \pi_{F}\right)\left(\beta\left(\varepsilon_{t}, \Delta_{F}\right)-\varepsilon_{t}\right), \alpha_{F, k}\right\rangle
$$

changes as $\varepsilon_{t}$ crosses the wall $E$, exactly when $\Delta_{F} \cap \partial P$ is contained in $E$ and $\alpha_{F, k}$ is $j$-perpendicular to $\pi_{F}(E)$. Hence, if $\operatorname{dim} F \neq d-1$, this sign changes if and only if $\Delta_{F}$ is contained in (exactly) one affine space $\Delta_{\widetilde{F}}$ perpendicular to $E$ with $\widetilde{F}$ a face of $\mathbf{C}_{\sigma_{i}(F)}$ with $\operatorname{dim} \widetilde{F}=\operatorname{dim} F+1$. Indeed, $\Delta_{\widetilde{F}}$ is such that

$$
\pi_{F}\left(\Delta_{\widetilde{F}}\right)=\pi_{F}\left(\Delta_{F}\right)+\mathbb{R} \alpha_{F, k}
$$

and unicity follows from dimensional reasons. On the other hand, if $\operatorname{dim} F=$ $d-1$, the sign of (17) changes if and only if $\Delta_{F} \cap P \subseteq E$. In this case, we define $\widetilde{F}$ to be $P$, and so $\operatorname{dim} \widetilde{F}=\operatorname{dim} F+1$ and $\Delta_{F}$ is contained in $\Delta_{\widetilde{F}}=V$.

In all cases, if $F$ is a simple face of $P$, then $\widetilde{F}$ is also a simple face of $P$ and so, from the proof of Theorem 5.1 presented in [3], one knows that the differences between the contributions of $F$ to the RHS of (16) before and after $\varepsilon_{t}$ crosses the wall, and those of $\widetilde{F}$, sum to zero.

Let us then consider the case where $F$ is a non-simple face of $P$ and let us assume, without loss of generality, that the sign of (17) flips from negative to positive as $\varepsilon_{t}$ crosses the wall $E$. Let $\mathcal{T}_{F}=\left\{\sigma_{1}(F), \ldots, \sigma_{L_{F}}(F)\right\}$ be the restriction of $\mathcal{T}$ to $\mathbf{N}_{F}$ (with $L_{F}=r_{F}-(\operatorname{codim} F-1)$ ) giving the simple cones $\mathbf{C}_{\sigma_{i}(F)}$. If the sign of $\left\langle\left(j^{-1} \circ \pi_{F}\right)\left(\beta\left(\varepsilon_{t}, \Delta_{F}\right)-\varepsilon_{t}\right), \alpha_{F, k}\right\rangle$ changes as $\varepsilon_{t}$ crosses a wall then

$$
\begin{aligned}
& \left.\left(\mathbf{1}_{\mathcal{T}_{F}}^{w}\right)^{1}:=\sum_{i}(-1)^{\left(m_{F, i}\right)^{1}} \mathbf{1}_{\left(\mathbf{C}_{\sigma_{i}(F)}^{w}\right.}^{w}\right)^{1} \\
& =\sum_{\substack{i \text { s.t. } \pm \alpha_{F, k} \text { is not } \\
\text { a gen. of the transverse } \\
\text { cone of }\left(\mathbf{C}_{\sigma_{i}(F)}^{\sharp}\right)^{1}}}(-1)^{\left(m_{F, i}\right)^{1}} \mathbf{1}_{\left(\mathbf{C}_{\sigma_{i}(F)}^{\sharp}\right)^{1}} \\
& \left.+\sum_{i \text { s.t. } \pm \alpha_{F, k} \text { is }}(-1)^{\left(m_{F, i}\right)^{1}} \mathbf{1}_{\left(\mathbf{C}_{\sigma_{i}(F)}^{\sharp}\right.}^{w}\right)^{1} \\
& \text { a gen. of the transverse } \\
& \text { cone of }\left(\mathbf{C}_{\sigma_{i}(F)}^{\sharp}\right)^{1}
\end{aligned}
$$


and

$$
\begin{aligned}
& \left.\left(\mathbf{1}_{\mathcal{T}_{F}}^{w}\right)^{2}:=\sum_{i}(-1)^{\left(m_{F, i}\right)^{2}} \mathbf{1}_{\left(\mathbf{C}_{\sigma_{i}(F)}^{\sharp}\right.}\right)^{2} \\
& =\sum_{i \text { s.t. } \pm \alpha_{F, k} \text { is not }}(-1)^{\left(m_{F, i}\right)^{1}} \mathbf{1}_{\left(\mathbf{C}_{\sigma_{i}(F)}^{\sharp}\right)^{2}} \\
& \text { a gen. of the transverse } \\
& \text { cone of }\left(\mathbf{C}_{\sigma_{i}(F)}^{\sharp}\right)^{2} \\
& -\sum_{i \text { s.t. } \pm \alpha_{F, k} \text { is }}(-1)^{\left(m_{F, i}\right)^{1}} \mathbf{1}_{\left(\mathbf{C}_{\sigma_{i}(F)}^{\sharp}\right)^{2}}, \\
& \text { a gen. of the transverse } \\
& \text { cone of }\left(\mathbf{C}_{\sigma_{i}(F)}^{\sharp}\right)^{2}
\end{aligned}
$$

where $\left(\mathbf{C}_{\sigma_{i}(F)}^{\sharp}\right)^{1},\left(\mathbf{C}_{\sigma_{i}(F)}^{\sharp}\right)^{2}$ are the polarized cones before and after the crossing.

Now one has to consider two cases. If $\pm \alpha_{F, k}$ is a generator of the transverse cone $\mathbf{T}_{F}$ along $F$ (see Figure 5 for the case where $\operatorname{dim} F=0$ ), let $I_{k} \subset\left\{1, \ldots, L_{F}\right\}$ be the index set of the cones $\mathbf{C}_{\sigma_{i}(F)}^{\sharp}$ whose transverse cones have $\pm \alpha_{F, k}$ as a generator. The difference between the contributions of $F$ to the RHS of (16) before and after $\varepsilon_{t}$ crosses the wall is then

$$
\sum_{i \in I_{k}}(-1)^{\left(m_{F, i}\right)^{1}}\left(\mathbf{1}_{\left(\mathbf{C}_{\sigma_{i}(F)}^{\sharp}\right)^{1}}^{w}+\mathbf{1}_{\left(\mathbf{C}_{\sigma_{i}(F)}^{\sharp}\right)^{2}}^{w}\right)= \pm \mathbf{1}_{\mathbf{C}_{\widetilde{F}}^{\sharp},}^{w},
$$

where $\widetilde{F}$ is a common face of all $\mathbf{C}_{\sigma_{i}(F)}^{\sharp}$ with $i \in I_{k}$ (and of $P$ ) such that

$$
\pi_{F}(\widetilde{F})=\pi_{F}\left(\Delta_{F}\right)+\mathbb{R} \alpha_{F, k}
$$

and where $\mathbf{C}_{\widetilde{F}}^{\sharp}$ is the polarized tangent cone to $P$ at $\widetilde{F}$. (Note that $\left|I_{k}\right|=r_{\widetilde{F}}-$ (codim $\widetilde{F}-1$ ) since, if $\widetilde{F}$ is $\underset{\widetilde{F}}{\text { non-simple, by compatibility of the triangulations }}$ used for $F$ and $\widetilde{F}$, the face $\widetilde{F}$ has to be contained in exactly $r_{\widetilde{F}}-(\operatorname{codim} \widetilde{F}-$ 1) cones $\mathbf{C}_{\sigma_{i}(F)}^{\sharp}$. $)$ On the other hand, $\beta\left(\varepsilon_{1}, \Delta_{\widetilde{F}}\right) \in P$, while $\beta\left(\varepsilon_{2}, \Delta_{\widetilde{F}}\right) \notin P$ and so the corresponding contributions of $\widetilde{F}$ to the right hand side of (16) are

$$
\mp \mathbf{1}_{\mathbf{C}_{\widetilde{F}}^{\sharp}}^{w} \quad \text { and } \quad 0 .
$$

The $\mp$ sign in $\mp \mathbf{1}_{\mathbf{C}_{\widetilde{F}}^{\sharp}}^{w}$ follows from the fact that the vectors

$$
v_{i}:=\pi_{F}\left(\beta\left(\beta\left(\varepsilon_{i}, \Delta_{\widetilde{F}}\right), \Delta_{F}\right)\right)-\pi_{F}\left(\beta\left(\varepsilon_{i}, \Delta_{\widetilde{F}}\right)\right) \quad(i=1,2)
$$


are of the form $v_{i}=r_{i} \alpha_{F, k}$, with $r_{i} \in \mathbb{R}$ satisfying $r_{1}<0$ and $r_{2}>0$, since

$$
\begin{aligned}
\left\langle j^{-1}\left(v_{i}\right), \alpha_{F, k}\right\rangle= & \left\langle j^{-1}\left(\varepsilon_{i}\right)-j^{-1}\left(\beta\left(\varepsilon_{i}, \Delta_{\widetilde{F}}\right)\right), \alpha_{F, k}\right\rangle \\
& +\left\langle j^{-1}\left(\beta\left(\varepsilon_{i}, \Delta_{F}\right)\right)-j^{-1}\left(\varepsilon_{i}\right), \alpha_{F, k}\right\rangle \\
= & \left\langle j^{-1}\left(\beta\left(\varepsilon_{i}, \Delta_{F}\right)\right)-j^{-1}\left(\varepsilon_{i}\right), \alpha_{F, k}\right\rangle .
\end{aligned}
$$

Consequently, the differences in the contributions of $F$ to the RHS of (16) before and after $\varepsilon_{t}$ crosses the wall, and those of $\widetilde{F}$, sum to zero (cf. Figure 5 ).

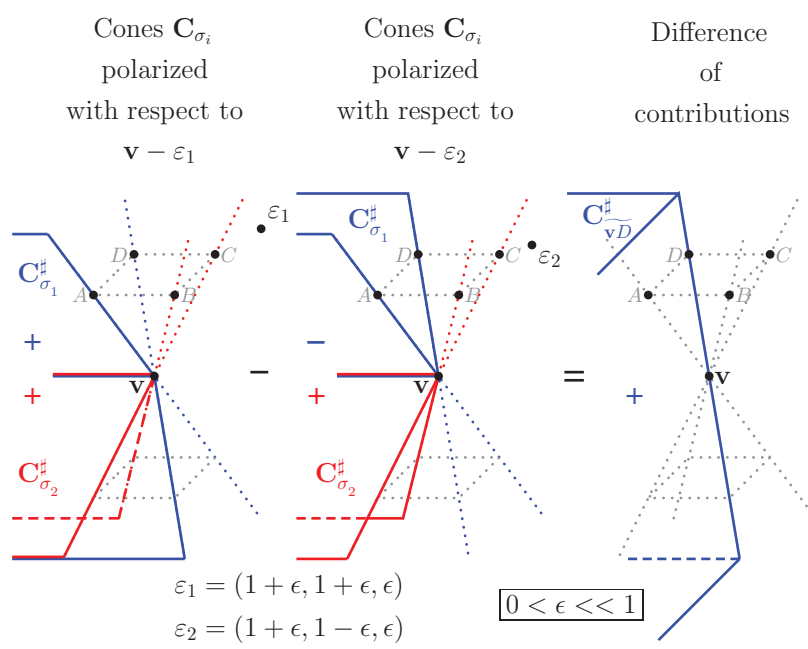

Figure 5: Polarized cones when the generator is an edge vector.

If, however, $\pm \alpha_{F, k}$ is not a generator of the transverse cone along $F$ (cf. Figure 6) then there exist exactly two simple cones, say $\mathbf{C}_{\sigma_{i_{A}}(F)}$ and $\mathbf{C}_{\sigma_{i_{B}}(F)}$ (with $\sigma_{i_{A}}(F), \sigma_{i_{B}}(F)$ in $\mathcal{T}_{F}$ ), whose transverse cones have $\pm \alpha_{F, k}$ as generator. Moreover, if for a given $\varepsilon$, the vector $\pm \alpha_{F, k}$ is a generator of the transverse cone of $\mathbf{C}_{\sigma_{i_{A}}(F)}$, then $\mp \alpha_{F, k}$ is a generator of the transverse cone of $\mathbf{C}_{\sigma_{i_{B}}(F)}$. Hence, $(-1)^{m_{F, i_{A}}}=-(-1)^{m_{F, i_{B}}}$ for both $\varepsilon_{1}$ and $\varepsilon_{2}$. Consequently, the difference in the contributions of $F$ to (16) before and after $\varepsilon_{t}$ crosses 
the wall is

$$
\begin{aligned}
& \sum_{i \text { s.t. } \pm \alpha_{F, k} \text { is }}(-1)^{\left(m_{F, i}\right)^{1}}\left(\mathbf{1}_{\left(\mathbf{C}_{\sigma_{i}(F)}^{\sharp}\right)^{1}}^{w}+\mathbf{1}_{\left(\mathbf{C}_{\sigma_{i}(F)}^{\sharp}\right)^{2}}^{w}\right) \\
& \text { a gen. of the transverse } \\
& \text { cone of } \mathbf{C}_{\sigma_{i}(F)} \\
& =(-1)^{\left(m_{F, i_{A}}\right)^{1}}\left(\mathbf{1}_{\left(\mathbf{C}_{\sigma_{i_{A}}(F)}\right)^{1}}+\mathbf{1}_{\left(\mathbf{C}_{\sigma_{i_{A}}(F)}^{\sharp}\right)^{2}}^{w}\right) \\
& +(-1)^{\left(m_{F, i_{B}}\right)^{1}}\left(\mathbf{1}_{\left(\mathbf{C}_{\sigma_{i_{B}}(F)}\right.}^{w}+\mathbf{1}_{\left(\mathbf{C}_{\sigma_{i_{B}}(F)}^{\sharp}\right)^{2}}^{w}\right) \\
& =(-1)^{\left(m_{F, i_{A}}\right)^{1}}\left(\mathbf{1}_{\mathbf{C}_{\widetilde{F}}^{\sharp}}^{w}-\mathbf{1}_{\mathbf{C}_{\widetilde{F}}^{\sharp}}^{w}\right)=0,
\end{aligned}
$$

where $\widetilde{F}$ is the face of $\mathbf{C}_{\sigma_{i_{A}}(F)}^{\sharp}\left(\right.$ or $\left.\mathbf{C}_{\sigma_{i_{B}}(F)}^{\sharp}\right)$ with $\operatorname{dim} \widetilde{F}=\operatorname{dim} F+1$, such that

$$
\pi_{F}(\widetilde{F})=\pi_{F}\left(\Delta_{F}\right)+\mathbb{R} \alpha_{F, k},
$$

and where $\mathbf{C}_{\widetilde{F}}^{\sharp}$ is the polarized tangent cone to $\mathbf{C}_{\sigma_{i_{A}}(F)}\left(\right.$ or $\left.\mathbf{C}_{\sigma_{i_{B}}(F)}\right)$ at $\widetilde{F}$ (cf. Figure 6). Note that the union of the cones $\left(\mathbf{C}_{\sigma_{i_{A}}(F)}^{\sharp}\right)^{1}$ and $\left(\mathbf{C}_{\sigma_{i_{A}}(F)}^{\sharp}\right)^{2}$ and the union of the cones $\left(\mathbf{C}_{\sigma_{i_{B}}(F)}^{\sharp}\right)^{1}$ and $\left(\mathbf{C}_{\sigma_{i_{B}}(F)}^{\sharp}\right)^{2}$ are both equal to $\mathbf{C}_{\widetilde{F}}^{\sharp}$.

Cones $\mathbf{C}_{\sigma_{i}} \quad$ Cones $\mathbf{C}_{\sigma_{i}} \quad$ Difference of contributions

polarized polarized

with respect to with respect to

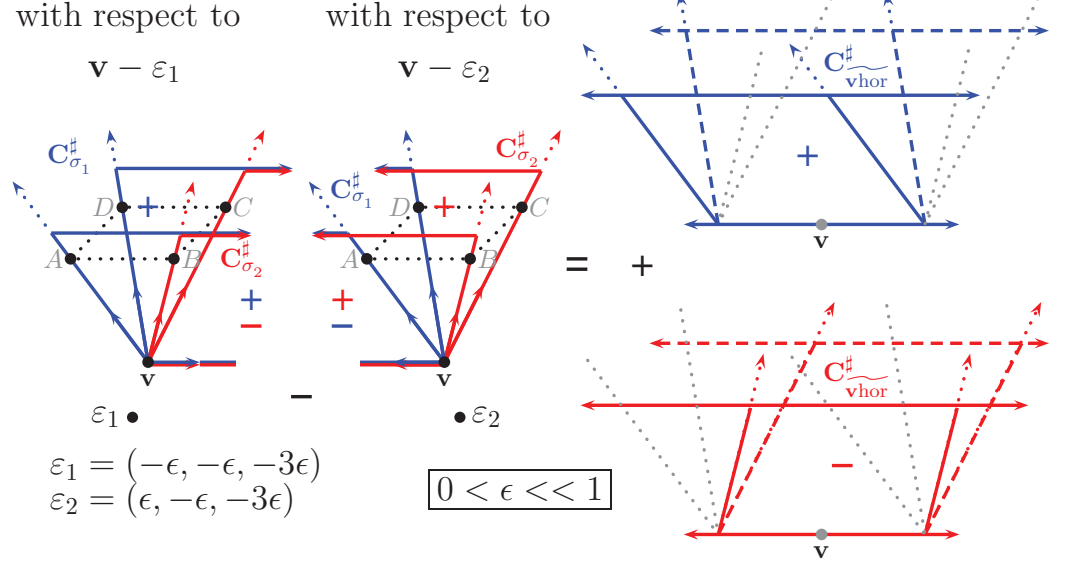

Figure 6: Polarized cones when the generator is not an edge vector.

Finally, we note that, if for a given face $\widetilde{F}$ of $P$, the value of $\mathbf{1}_{P}\left(\beta\left(\varepsilon_{t}, \Delta_{\widetilde{F}}\right)\right)$ changes when crossing the wall $E$, the intersection of $\Delta_{\widetilde{F}}$ with $E$ contains 
$\Delta_{F} \cap P$ for (exactly) one face $F$ of $P$ with $\operatorname{dim} F=\operatorname{dim} \widetilde{F}-1$, and the result then follows.

Step 2: First we take an $\varepsilon \in W$ for which $\beta\left(\varepsilon, \Delta_{F}\right) \notin P$, whenever $F$ is a face with $\operatorname{dim} F>0$; that is, we take

$$
\varepsilon \in \widetilde{W}:=\bigcap_{\substack{F \text { face of } P \text { s.t. } \\ \operatorname{dim} F>0}}\left\{\mathbf{x} \in V \mid \beta\left(\mathbf{x}, \Delta_{F}\right) \cap F=\varnothing\right\}
$$

Then the equality in (16) becomes

$$
\mathbf{1}_{P}^{w}(\mathbf{x})=\sum_{\mathbf{v} \text { vertex of } P} \mathbf{1}_{\mathcal{T}_{\mathbf{v}}}^{w}(\mathbf{x})
$$

To prove (19) we choose a vector $\xi \in V^{*}$ for which (19) becomes a weighted version of the Lawrence-Varchenko decomposition for non-simple polytopes, where the cones involved are polarized with respect to $\xi$. The existence of such a polarizing vector can be shown as in the proof of Theorem 5.1 in [3]. Note that this Lawrence-Varchenko decomposition is a weighted version of the one shown in [9]. Nevertheless, for $\mathbf{x} \in V \backslash S$, it follows easily from Lemma 6.5 below, once we know that the sum

$$
\sum_{\mathbf{v} \text { vertex of } P} \mathbf{1}_{\mathcal{T}_{\mathbf{v}}}^{w}
$$

where all the cones involved are polarized with respect to a fixed vector $\xi$, is independent of the choice of polarizing vector (which we know by Step 1 as explained in Remark 6.7). The proof goes as follows.

For $\mathbf{x} \in P \backslash S$ we consider the (simple) face $F_{\mathbf{x}}$ of $P$ such that $\mathbf{x} \in \operatorname{int} F_{\mathbf{x}}$ and we pick a vertex $\mathbf{v}_{0}$ in this face. If $\mathbf{v}_{0}$ is a simple vertex, then we proceed as in $[3,13]$ and we are done. If, however, $\mathbf{v}_{0}$ is a non-simple vertex, we consider the cones $\mathbf{C}_{\sigma_{1}\left(\mathbf{v}_{0}\right)}, \ldots, \mathbf{C}_{\sigma_{L_{\mathbf{v}}}\left(\mathbf{v}_{0}\right)}$ (with vertex $\mathbf{v}_{0}$ ) defined by the triangulation $\mathcal{T}_{\mathbf{v}_{0}}$, and we take $i_{0} \in\left\{1, \ldots, L_{\mathbf{v}_{0}}\right\}$ for which $F_{\mathbf{x}}$ is contained in a face of $\mathbf{C}_{\sigma_{i_{0}}\left(\mathbf{v}_{0}\right)}$ of the same dimension. Since the sum in (20) does not depend on the polarizing vector, we take a vector $\widetilde{\xi} \in V^{*}$ for which $\mathbf{C}_{\mathbf{v}_{0}}^{\sharp}=\mathbf{C}_{\mathbf{v}_{0}}$ and $\mathbf{C}_{\sigma_{i_{0}}\left(\mathbf{v}_{0}\right)}^{\sharp}=\mathbf{C}_{\sigma_{i_{0}}\left(\mathbf{v}_{0}\right)}$ (cf. Lemma 6.5 for the existence of this vector). Note that for every other vertex $\mathbf{v} \neq \mathbf{v}_{0}$ of $P$, we have $\mathbf{C}_{\mathbf{v}}^{\sharp} \neq \mathbf{C}_{\mathbf{v}}$. 
Then, at $\mathbf{x}$, the RHS of (19) is equal to

$$
\begin{aligned}
\sum_{\substack{\text { vvertex } \\
\text { of } P}} \mathbf{1}_{\mathcal{T}_{\mathbf{v}}}^{w}(\mathbf{x}) & =\mathbf{1}_{\mathcal{T}_{\mathbf{v}_{0}, \mathbf{v}_{0}}}^{w}(\mathbf{x})=\sum_{i=1}^{L_{\mathbf{v}_{0}}}(-1)^{m_{i, \mathbf{v}_{0}}} \mathbf{1}_{\mathbf{C}_{\sigma_{i}\left(\mathbf{v}_{0}\right)}^{\sharp}}^{w}(\mathbf{x}) \\
& =\mathbf{1}_{\mathbf{C}_{\sigma_{i_{0}}\left(\mathbf{v}_{0}\right)}}^{w}(\mathbf{x})=\prod_{j \in I_{F_{\mathbf{x}}}} q_{j}=\mathbf{1}_{P}^{w}(\mathbf{x}) .
\end{aligned}
$$

If $\mathbf{x} \in P^{c} \backslash S$ so that the LHS of (19) is zero, we take a vertex $\mathbf{v}_{1}$ of $P$ that minimizes the distance to $\mathbf{x}$. Again, if $\mathbf{v}_{1}$ is a simple vertex, we proceed as in $[3,13]$ and we are done. If $\mathbf{v}_{1}$ is a non-simple vertex, let $\mathbf{C}_{\sigma_{1}\left(\mathbf{v}_{1}\right)}, \ldots, \mathbf{C}_{\sigma_{L_{\mathbf{v}}}\left(\mathbf{v}_{1}\right)}$ be the simple cones (with vertex $\mathbf{v}_{1}$ ) defined by the triangulation $\mathcal{T}_{\mathbf{v}_{1}}$. Since $\mathbf{x} \notin \mathbf{C}_{\mathbf{v}_{1}}$ there exists an $i_{1} \in\left\{1, \ldots, L_{\mathbf{v}_{1}}\right\}$ such that $\mathbf{x} \notin \mathbf{C}_{\sigma_{i_{1}}\left(\mathbf{v}_{1}\right)}$ (because $\mathbf{C}_{\mathbf{v}_{1}}$ is equal to the intersection of the cones $\left.\mathbf{C}_{\sigma_{i}\left(\mathbf{v}_{1}\right)}\right)$. Choosing a polarizing vector $\widetilde{\xi}$ for which $\mathbf{C}_{\mathbf{v}_{1}}^{\sharp}=\mathbf{C}_{\mathbf{v}_{1}}$ and $\mathbf{C}_{\sigma_{i_{1}}\left(\mathbf{v}_{1}\right)}^{\sharp}=\mathbf{C}_{\sigma_{i_{1}}\left(\mathbf{v}_{1}\right)}$ (cf. Lemma 6.5), the RHS of (19) becomes equal to zero (since none of the polarized cones defined by the triangulations $\mathcal{T}_{\mathbf{v}}$ for the different vertices $\mathbf{v}$ of $P$ contains $\mathbf{x}$ ) and the result follows. (Note that all cones $\mathbf{C}_{\sigma_{i}\left(\mathbf{v}_{1}\right)}^{\sharp}$, for $i \neq i_{1}$ are contained in $\mathbf{C}_{\sigma_{i_{1}}\left(\mathbf{v}_{1}\right)}^{\sharp}$.)

Let us now see what happens when all the weights $q_{i}$ are equal to some fixed value $q \in \mathbb{C}$ and $\mathbf{x} \in S$. Assume first that $\mathbf{x} \in S \cap P$ and let $F_{\mathbf{x}}$ be the (non-simple) face of $P$ such that $\mathbf{x} \in \operatorname{int} F$. Let $\mathbf{v}_{0}$ be a vertex of $P$ in $F_{\mathbf{x}}$ and consider a cone $\mathbf{C}_{\sigma_{i_{0}}\left(\mathbf{v}_{0}\right)}$ defined by the triangulation $\mathcal{T}_{\mathbf{v}_{0}}$, with $F_{\mathbf{x}}$ contained in a face of $\mathbf{C}_{\sigma_{i_{0}}\left(\mathbf{v}_{0}\right)}$ of the same dimension. Using independence on the polarizing vector, we can take $\widetilde{\xi}$ such that $\mathbf{C}_{\mathbf{v}_{0}}^{\sharp}=\mathbf{C}_{\mathbf{v}_{0}}$ and $\mathbf{C}_{\sigma_{i_{0}}\left(\mathbf{v}_{0}\right)}^{\sharp}=\mathbf{C}_{\sigma_{i_{0}}\left(\mathbf{v}_{0}\right)}$ (cf. Lemma 6.5). Then, at $\mathbf{x}$, the RHS of (19) is equal to

$$
\sum_{\mathbf{v} \text { vertex of } P} \mathbf{1}_{\mathcal{T}_{\mathbf{v}}}^{w}(\mathbf{x})=\mathbf{1}_{\mathcal{T}_{\mathbf{v}_{0}}}^{w}(\mathbf{x})=\sum_{i=1}^{L_{\mathbf{v}_{0}}}(-1)^{m_{i, \mathbf{v}_{0}}} \mathbf{1}_{\mathbf{C}_{\sigma_{i}\left(\mathbf{v}_{0}\right)}^{\sharp}}^{w}(\mathbf{x}) .
$$

For each $i \neq i_{0}$, let $F_{i}$ be the facet of the polarized cone $\mathbf{C}_{\sigma_{i}\left(\mathbf{v}_{0}\right)}^{\sharp}$ that intersects $\mathbf{C}_{\mathbf{v}_{0}} \cap$ int $\mathbf{C}_{\sigma_{i_{0}}\left(\mathbf{v}_{0}\right)}^{\sharp}$ given by Lemma 6.5 . Then, since the cone $\mathbf{C}_{\sigma_{i_{0}}\left(\mathbf{v}_{0}\right)}^{\sharp}$ is simple, $F_{\mathbf{x}}$ must also be contained in the boundary of $\left(r_{F_{\mathbf{x}}}-\operatorname{codim} F_{\mathbf{x}}\right)$ other cones $\mathbf{C}_{\sigma_{i}\left(\mathbf{v}_{0}\right)}^{\sharp}$ with $i \neq i_{0}$ and so the sum in (21) equals

$$
q^{\operatorname{codim} F_{\mathbf{x}}}-\left(r_{F_{\mathbf{x}}}-\operatorname{codim} F_{\mathbf{x}}\right) q^{\operatorname{codim} F_{\mathbf{x}}-1}(1-q) .
$$

Indeed, $F_{\mathbf{x}}$ is an intersection of $m:=\operatorname{codim} F_{\mathbf{x}}$ facets of $\mathbf{C}_{\sigma_{i_{0}}\left(\mathbf{v}_{0}\right)}^{\sharp}$ (with weight $q)$ and, for each $\mathbf{C}_{\sigma_{i}\left(\mathbf{v}_{0}\right)}^{\sharp}$ with $i \neq i_{0}$ that it intersects, $F_{x}$ is the intersection of 
the facet $F_{i}$ (with weight $(1-q)$ ) with $m-1$ other facets of $\mathbf{C}_{\sigma_{i}\left(\mathbf{v}_{0}\right)}^{\sharp}$ (these cones are simple).

If $\mathbf{x} \in S \cap P^{c}$ we proceed similarly.

Finally, we state and prove the technical Lemma used in the proof above.

Lemma 6.5. Let $\mathbf{v}$ be a vertex of a convex polytope $P \subset V$ and let

$$
\mathcal{T}_{\mathbf{v}}=\left\{\sigma_{1}(\mathbf{v}), \ldots, \sigma_{L_{\mathbf{v}}}(\mathbf{v})\right\}
$$

be any regular triangulation of the inner normal cone $\mathbf{N}_{\mathbf{v}}$. Let $\xi \in V^{*}$ be a polarizing vector such that $\mathbf{C}_{\mathbf{v}}=\mathbf{C}_{\mathbf{v}}^{\sharp}$. Then, there exists exactly one $i_{0} \in$ $\left\{1, \ldots, L_{\mathbf{v}}\right\}$ such that

$$
\mathbf{C}_{\sigma_{i_{0}}(\mathbf{v})}^{\sharp}=\mathbf{C}_{\sigma_{i_{0}}(\mathbf{v})}
$$

and each of the other cones $\mathbf{C}_{\sigma_{i}(\mathbf{v})}^{\sharp}$ defined by $\mathcal{T}_{\mathbf{v}}$ intersects $\mathbf{C}_{\mathbf{v}} \cap \operatorname{int}\left(\mathbf{C}_{\sigma_{i_{0}}(\mathbf{v})}^{\sharp}\right)$ in (exactly) one facet $F_{i}$ of $P$ with $F_{i} \neq F_{j}$ if $i \neq j$.

Moreover, for every $i \in\left\{1, \ldots, L_{\mathbf{v}}\right\}$ there exists a polarizing vector $\xi \in$ $V^{*}$ for which $\mathbf{C}_{\mathbf{v}}=\mathbf{C}_{\mathbf{v}}^{\sharp}$ and $\mathbf{C}_{\sigma_{i}(\mathbf{v})}^{\sharp}=\mathbf{C}_{\sigma_{i}(\mathbf{v})}$.

Proof. Let $\mathbf{1}_{\mathcal{T}_{\mathbf{v}}, \mathbf{v}}$ be the (unweighted) function obtained from $\mathbf{1}_{\mathcal{T}_{\mathbf{v}}, \mathbf{v}}^{w}$ by making all the weights $q_{i}$ equal to 1 . Then,

$$
\mathbf{1}_{\mathcal{T}_{\mathbf{v}}}=\sum_{i=1}^{L_{\mathbf{v}}}(-1)^{m_{\mathbf{v}, i}} \mathbf{1}_{\mathbf{C}_{\sigma_{i}(\mathbf{v})}^{\sharp}}
$$

(note that we know from [9] that $\mathbf{1}_{\mathcal{T}_{\mathbf{v}}, \mathbf{v}}$ does not depend on the choice of a regular triangulation of the normal cone to $P$ at $\mathbf{v}$ ). For each facet $F$ of the polarized cones $\mathbf{C}_{\sigma_{i}(\mathbf{v})}^{\sharp}$, let $H_{F, i}$ be the halfspace bounded by $\Delta_{F}$ that contains $\mathbf{C}_{\sigma_{i}(\mathbf{v})}^{\sharp}$, and consider $H_{F, i}^{+}:=H_{F, i} \backslash \Delta_{F}$. Then, by [9], since we are using a polarizing vector for which $\mathbf{C}_{\mathbf{v}}{ }^{\sharp}=\mathbf{C}_{\mathbf{v}}$, we have

$$
\begin{aligned}
1=\mathbf{1}_{P}(\mathbf{v}) & =\mathbf{1}_{\mathcal{T}_{\mathbf{v}}}(\mathbf{v})=\sum_{i=1}^{L_{\mathbf{v}}}(-1)^{m_{\mathbf{v}, i}} \mathbf{1}_{\mathbf{C}_{\sigma_{i}(\mathbf{v})}^{\sharp}}(\mathbf{v})=\sum_{\substack{i \text { s.t. } H_{F, i}^{+} \cap P \neq \varnothing \\
\text { for all facets } F \text { of } \mathbf{C}_{\sigma_{i}(\mathbf{v})}^{\sharp}}} 1 \\
& =\#\left\{i \in\left\{1, \ldots, L_{\mathbf{v}}\right\} \mid H_{F, i}^{+} \cap P \neq \varnothing \text { for all facets of } \mathbf{C}_{\sigma_{i}(\mathbf{v})}^{\sharp}\right\} .
\end{aligned}
$$

Indeed, $\mathbf{1}_{\mathbf{C}_{\sigma_{i}(\mathbf{v})}^{\sharp}}(\mathbf{v})=1$ if, for all facets $F$ of $\mathbf{C}_{\sigma_{i}(\mathbf{v})}^{\sharp}$, we have $H_{F, i}^{+} \cap P \neq \varnothing$, and is zero otherwise. We conclude then that there must exist exactly one 
cone $\mathbf{C}_{\sigma_{i_{0}}(\mathbf{v})}^{\sharp}$ such that $H_{F, i_{0}}^{+} \cap P \neq \varnothing$ for all facets $F$ of $\mathbf{C}_{\sigma_{i_{0}}(\mathbf{v})}^{\sharp}$; i.e., such that $\mathbf{C}_{\sigma_{i_{0}}(\mathbf{v})}^{\sharp}=\mathbf{C}_{\sigma_{i_{0}}(\mathbf{v})}$. Hence all the other polarized cones $\mathbf{C}_{\sigma_{i}(\mathbf{v})}^{\sharp}$ with $i \neq i_{0}$ have at least one facet $F$ for which

$$
H_{F, i}^{+} \cap P=\varnothing
$$

and so they do not intersect the interior of $P$. By convexity, each of these polarized cones $\mathbf{C}_{\sigma_{i}(\mathbf{v})}^{\sharp}$ intersects $\mathbf{C}_{\mathbf{v}} \cap$ int $\mathbf{C}_{\sigma_{i_{0}}(\mathbf{v})}^{\sharp}$ in at most one facet of $\mathbf{C}_{\mathbf{v}}$. Moreover, $\mathbf{C}_{\mathbf{v}}$ has $r_{\mathbf{v}}$ facets and $d$ of these are contained in facets of $\mathbf{C}_{\sigma_{i_{0}}(\mathbf{v})}^{\sharp}$. Since every triangulation of $\mathbf{N}_{\mathbf{v}}$ generates $r_{\mathbf{v}}-(d-1)$ simple cones with vertex $\mathbf{v}$, each cone $\mathbf{C}_{\sigma_{i}(\mathbf{v})}^{\sharp}$, with $i \neq i_{0}$, intersects $\mathbf{C}_{\mathbf{v}} \cap \operatorname{int}\left(\mathbf{C}_{\sigma_{i_{0}}(\mathbf{v})}^{\sharp}\right)$ in a different facet $F_{i}$ of $\mathbf{C}_{\mathbf{v}}$. For all other facets $F$ of $\mathbf{C}_{\sigma_{i}(\mathbf{v})}^{\sharp}$ with $F \neq F_{i}$ we have by convexity that

$$
H_{F, i}^{+} \cap P \neq \varnothing
$$

(cf. Figure 7). Note that all $\mathbf{C}_{\sigma_{i}(\mathbf{v})}^{\sharp}$ with $i \neq i_{0}$ are contained in $\mathbf{C}_{\sigma_{i_{0}}(\mathbf{v})}^{\sharp}$.

To show that for every $i \in\left\{1, \ldots, L_{\mathbf{v}}\right\}$ there exists a polarizing vector for which $\mathbf{C}_{\mathbf{v}}=\mathbf{C}_{\mathbf{v}}^{\sharp}$ and $\mathbf{C}_{\sigma_{i}(\mathbf{v})}^{\sharp}=\mathbf{C}_{\sigma_{i}(\mathbf{v})}$, we consider a half-space $H$ with $\mathbf{v} \in \partial H$ and $\mathbf{C}_{\sigma_{i}(\mathbf{v})} \subset H$ (and consequently $P \subset H$ since all cones $\mathbf{C}_{\sigma_{i}(\mathbf{v})}$ contain $P$ ), and take any inward pointing vector $\xi \in V^{*}$ orthogonal to the bounding hyperplane of $H$. Then $\langle\xi, \mathbf{v}\rangle \leq\langle\xi, \mathbf{y}\rangle$ for every $\mathbf{y} \in \mathbf{C}_{\sigma_{i}(\mathbf{v})}$ (and hence for every $\mathbf{y} \in \mathbf{C}_{\mathbf{v}}$ ), implying that $\mathbf{C}_{\sigma_{i}(\mathbf{v})}^{\sharp}=\mathbf{C}_{\sigma_{i}(\mathbf{v})}$ and $\mathbf{C}_{\mathbf{v}}^{\sharp}=\mathbf{C}_{\mathbf{v}}$.

We will now show that the functions $\mathbf{1}_{\mathcal{T}_{F}}^{w}$ do not depend on the choice of a regular triangulation of the normal cone at $F$. For that we first consider the case where $F$ is a vertex $\mathbf{v}$.

Proposition 6.6. Let $P \subset V$ be a convex polytope and let $\varepsilon \in W$. For a vertex $\mathbf{v}$ of $P$, let $S_{\mathbf{v}}$ be the union of the affine spaces generated by nonsimple faces of $P$ that contain $\mathbf{v}$. Then, the values of the weighted functions $\mathbf{1}_{\mathcal{T}_{\mathbf{v}}}^{w}$ on $V \backslash S_{\mathbf{v}}$, obtained from a regular triangulation $\mathcal{T}_{\mathbf{v}}$ of the normal cone to $P$ at $\mathbf{v}$, do not depend on the choice of triangulation.

Moreover, in the particular case where all the weights $q_{i}\left(i=1, \ldots, N_{P}\right)$ are equal, the values of $\mathbf{1}_{\mathcal{T}_{\mathbf{v}}}^{w}$ on the whole space $V$ do not depend on the choice of the regular triangulation.

Proof. Let $\mathbf{v}_{0}$ be a vertex of $P$ and consider $\xi:=j^{-1}\left(\beta_{\mathbf{v}_{0}}\right)=j^{-1}\left(\mathbf{v}_{0}-\varepsilon\right)$. Then take $\varepsilon^{\prime} \in W$ such that the polytope decomposition in Proposition 6.2 with respect to $\varepsilon^{\prime}$ is the Lawrence-Varchenko decomposition with respect to $\xi$ (cf. Remark 6.7). Let $\mathcal{T}_{\mathbf{v}_{0}}^{1}$ and $\mathcal{T}_{\mathbf{v}_{0}}^{2}$ be two regular triangulations of the 


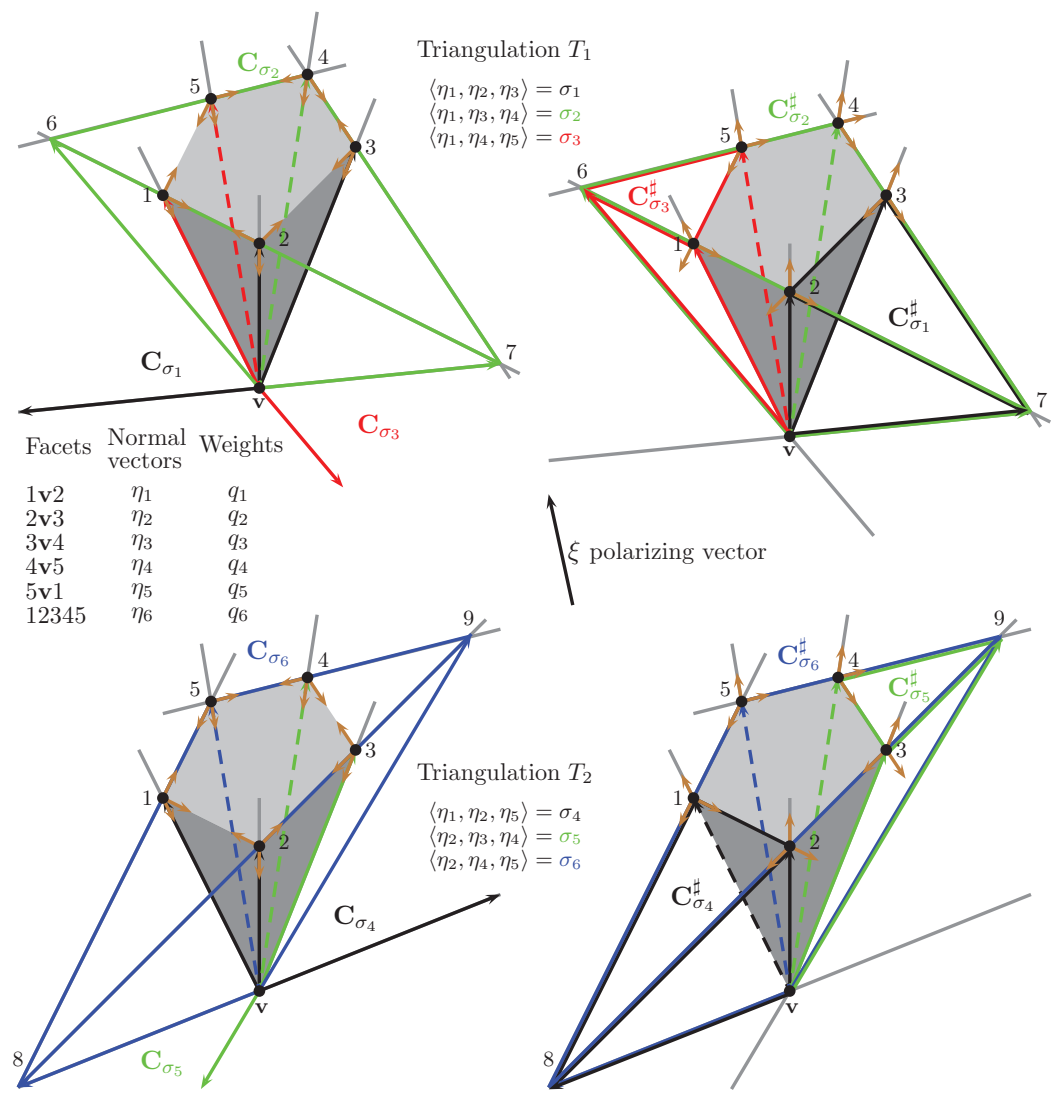

Figure 7: An Illustration of Lemma 6.5.

normal cone $\mathbf{N}_{\mathbf{v}_{0}}$ and let us choose two regular triangulations $\mathcal{T}^{1}, \mathcal{T}^{2}$ of the polar dual polytope $P^{*}$ that restrict to $\mathcal{T}_{\mathbf{v}_{0}}^{1}$ and $\mathcal{T}_{\mathbf{v}_{0}}^{2}$ on $\mathbf{N}_{\mathbf{v}_{0}}$, respectively. Then, for $\mathrm{x} \in V \backslash S$, it follows from Proposition 6.2 that

$$
\mathbf{1}_{P}^{w}(\mathbf{x})=\sum_{\mathbf{v} \text { vertex of } P} \mathbf{1}_{\mathcal{T}_{\mathbf{v}}^{1}, \mathbf{v}}^{w}(\mathbf{x})=\sum_{\mathbf{v} \text { vertex of } P} \mathbf{1}_{\mathcal{T}_{\mathbf{v}}^{2}, \mathbf{v}}^{w}(\mathbf{x}),
$$

where $\mathcal{T}_{\mathbf{v}}^{1}$ and $\mathcal{T}_{\mathbf{v}}^{2}$ are the restrictions of $\mathcal{T}^{1}$ and $\mathcal{T}^{2}$ to the different normal cones $\mathbf{N}_{\mathbf{v}}$. Just like in [9] the functions $\mathbf{1}_{\mathcal{T}_{\mathbf{v}}^{i}, \mathbf{v}}^{w}, i=1,2$, are both conic and positive, meaning that for every $\mathbf{w} \in V \backslash\{0\}, \mathbf{1}_{\mathcal{T}_{\mathbf{v}}^{i}}^{w}(\mathbf{v}+\lambda \mathbf{w})$ is constant in $\lambda>0$, and $\mathbf{1}_{\mathcal{T}_{\mathbf{v}}^{i}}^{w}(\mathbf{v}+\mathbf{w})=0$ if $\langle\xi, \mathbf{w}\rangle<0$. Hence, like in [9], we conclude that $\mathbf{1}_{\mathcal{T}_{\mathbf{v}}^{1}, \mathbf{v}}^{w}=\mathbf{1}_{\mathcal{T}_{\mathbf{v}}^{2}, \mathbf{v}}^{w}$ for every vertex $\mathbf{v}$ of $P$ and so, in particular, $\mathbf{1}_{\mathcal{T}_{\mathbf{v}_{0}}^{1}, \mathbf{v}_{0}}^{w}=\mathbf{1}_{\mathcal{T}_{\mathbf{v}_{0}}^{2}, \mathbf{v}_{0}}^{w}$. To see this, let us first order the vertices $\mathbf{v}_{1}, \ldots, \mathbf{v}_{k}$ of $P$ in increasing order 
of the value $\left\langle\xi, \mathbf{v}_{j}\right\rangle$ (just sweep $V$ by hyperplanes $\langle\xi, \mathbf{x}\rangle=c$ ). For $\mathbf{x} \in V$ such that $\langle\xi, \mathbf{x}\rangle<\left\langle\xi, \mathbf{v}_{1}\right\rangle$, we trivially have

$$
\mathbf{1}_{\mathcal{T}_{\mathbf{v}_{j}}^{1}, \mathbf{v}_{j}}^{w}(\mathbf{x})=\mathbf{1}_{\mathcal{T}_{\mathbf{v}_{j}}^{2}, \mathbf{v}_{j}}^{w}(\mathbf{x})=0, \text { for every } j=1, \ldots, k
$$

by positivity. For $\mathbf{x} \in V \backslash S_{\mathbf{v}_{1}}$ such that $\langle\xi, \mathbf{x}\rangle \in\left[\left\langle\xi, \mathbf{v}_{1}\right\rangle,\left\langle\xi, \mathbf{v}_{2}\right\rangle[\right.$ ( $\mathbf{x}$ is between two hyperplanes $\langle\xi, \mathbf{x}\rangle=c$ with $c=\left\langle\xi, \mathbf{v}_{1}\right\rangle$ and $\left.c=\left\langle\xi, \mathbf{v}_{2}\right\rangle\right)$, we have

$$
\begin{aligned}
\mathbf{1}_{\mathcal{T}_{\mathbf{v}_{1}}^{1}, \mathbf{v}_{1}}^{w} & (\mathbf{x})=\sum_{j=1}^{k} \mathbf{1}_{\mathcal{T}_{\mathbf{v}_{j}}^{1}, \mathbf{v}_{j}}^{w}(\mathbf{x}) \text { (since, by positivity, } \mathbf{1}_{\mathcal{T}_{\mathbf{v}_{j}}^{1}, \mathbf{v}_{j}}^{w}(\mathbf{x})=0 \text { for all } j>1 \text { ) } \\
& =\mathbf{1}_{P}^{w}(\mathbf{x})=\sum_{j=1}^{k} \mathbf{1}_{\mathcal{T}_{\mathbf{v}_{j}}^{2}, \mathbf{v}_{j}}^{w}(\mathbf{x})=\mathbf{1}_{\mathcal{T}_{\mathbf{v}_{1}}^{2}, \mathbf{v}_{1}}^{w}(\mathbf{x}) \text { (again by positivity) }
\end{aligned}
$$

and $\mathbf{1}_{\mathcal{T}_{\mathbf{v}_{j}}^{1}}^{w}(\mathbf{x})=\mathbf{1}_{\mathcal{T}_{\mathbf{v}_{j}}^{2}, \mathbf{v}_{1}}^{w}(\mathbf{x})=0$ for every $j \geq 2$ by positivity. We now proceed by induction: let $\mathbf{x} \in V \backslash S$ such that $\langle\xi, \mathbf{x}\rangle \in\left[\left\langle\xi, \mathbf{v}_{i}\right\rangle,\left\langle\xi, \mathbf{v}_{i+1}\right\rangle[\right.$, and assume that $\mathbf{1}_{\mathcal{T}_{\mathbf{v}_{j}}^{1}}^{w}(\mathbf{y})=\mathbf{1}_{\mathcal{T}_{\mathbf{v}_{j}}^{2}}^{w}(\mathbf{y})$ for every $\mathbf{y} \in\left[\left\langle\xi, \mathbf{v}_{j}\right\rangle,\left\langle\xi, \mathbf{v}_{j+1}\right\rangle[\right.$ with $j<i$,

$$
\begin{aligned}
& \left.\mathbf{1}_{\mathcal{T}_{\mathbf{v}_{i}}^{1}}^{w}(\mathbf{x})=\sum_{j=i}^{k} \mathbf{1}_{\mathcal{T}_{\mathbf{v}_{j}^{1}}^{1}}^{w}(\mathbf{x}) \quad \text { (since, by positivity, } \mathbf{1}_{\mathcal{T}_{\mathbf{v}_{j}}^{1}}^{w}(\mathbf{x})=0 \text { for } j>i\right) \\
= & \mathbf{1}_{P}^{w}(\mathbf{x})-\sum_{j=1}^{i-1} \mathbf{1}_{\mathcal{T}_{\mathbf{v}_{j}}^{1}}^{w}(\mathbf{x}) \\
= & \mathbf{1}_{P}^{w}(\mathbf{x})-\sum_{j=1}^{i-1} \mathbf{1}_{\mathcal{T}_{\mathbf{v}_{j}}^{1}}^{w}\left(\mathbf{x}_{j}\right) \quad \text { (for some } \mathbf{x}_{j} \text { s.t. }\left\langle\xi, \mathbf{x}_{j}\right\rangle \in\left[\left\langle\xi, \mathbf{v}_{j}\right\rangle,\left\langle\xi, \mathbf{v}_{j+1}\right\rangle[)\right. \\
= & \mathbf{1}_{P}^{w}(\mathbf{x})-\sum_{j=1}^{i-1} \mathbf{1}_{\mathcal{T}_{\mathbf{v}_{j}}^{2}}^{w}\left(\mathbf{x}_{j}\right)=\mathbf{1}_{P}^{w}(\mathbf{x})-\sum_{j=1}^{i-1} \mathbf{1}_{\mathcal{T}_{\mathbf{v}_{j}}}^{w}(\mathbf{x})=\sum_{j=i}^{k} \mathbf{1}_{\mathcal{T}_{\mathbf{v}_{j}}^{2}}^{w}(\mathbf{x}) \\
= & \mathbf{1}_{\mathcal{T}_{\mathbf{v}_{i}}^{2}}^{w}(\mathbf{x}) \quad(\text { again by positivity), }
\end{aligned}
$$

where the fact that the functions involved are conic allowed us to use $\mathbf{x}_{j}$ instead of $\mathbf{x}$. We conclude that

- $\mathbf{1}_{\mathcal{T}_{\mathbf{v}_{i}}^{1}}^{w}(\mathbf{x})=\mathbf{1}_{\mathcal{T}_{\mathbf{v}_{i}}^{2}}^{w}(\mathbf{x})$;

- $\mathbf{1}_{\mathcal{T}_{\mathbf{v}_{j}}^{1}}^{w}(\mathbf{x})=\mathbf{1}_{\mathcal{T}_{\mathbf{v}_{j}}^{2}}^{w}(\mathbf{x})$ for $j>i$, by positivity;

- $\mathbf{1}_{\mathcal{T}_{\mathbf{v}_{j}^{1}}^{1}}^{w}(\mathbf{x})=\mathbf{1}_{\mathcal{T}_{\mathbf{v}_{j}}^{1}}^{w}\left(\mathbf{x}_{j}\right)=\mathbf{1}_{\mathcal{T}_{\mathbf{v}_{j}}^{2}}^{w}\left(\mathbf{x}_{j}\right)=\mathbf{1}_{\mathcal{T}_{\mathbf{v}_{j}}^{2}}^{w}(\mathbf{x})$ for some $\mathbf{x}_{j} \in\left[\left\langle\xi, \mathbf{v}_{j}\right\rangle,\left\langle\xi, \mathbf{v}_{j+1}\right\rangle[\right.$ with $j<i$, by the fact that the functions involved are conic. 
Hence, for every $\mathbf{x} \in V \backslash S$ and every vertex $\mathbf{v}$ of $P$, we have $\mathbf{1}_{\mathcal{T}_{\mathbf{v}}^{1}}^{w}(\mathbf{x})=\mathbf{1}_{\mathcal{T}_{\mathbf{v}}}^{w}(\mathbf{x})$ and, in particular, $\mathbf{1}_{\mathcal{T}_{\mathbf{v}_{0}}^{1}, \mathbf{v}_{0}}^{w}=\mathbf{1}_{\mathcal{T}_{\mathbf{v}_{0}}, \mathbf{v}_{0}}^{w}$ on $V \backslash S$ and consequently on $V \backslash S_{\mathbf{v}_{0}}$.

We proceed similarly for the case where all the weights $q_{i}$ are equal to some fixed value $q \in \mathbb{C}$, taking now $\mathbf{x} \in V$ and the function $f: V \rightarrow \mathbb{C}$ given by

$$
f(\mathbf{x})= \begin{cases}\mathbf{1}_{P}^{w}(\mathbf{x}) & \text { if } \mathbf{x} \in P \backslash S \\ q^{\operatorname{codim} F_{\mathbf{x}}}-\left(r_{F_{\mathbf{x}}}-\operatorname{codim} F_{\mathbf{x}}\right) q^{\operatorname{codim} F_{\mathbf{x}}-1}(1-q) & \text { if } \mathbf{x} \in P \cap S, \\ 0 & \text { otherwise }\end{cases}
$$

instead of $\mathbf{1}_{P}^{w}$.

Remark 6.7. In the above proofs we have used several times the fact that, given a polarizing vector $\xi$, we can always take $\varepsilon>0$ for which the decomposition in Proposition 6.2 becomes the Lawrence-Varchenko decomposition with respect to $\xi$. Let us see how this works. For every polarization there exists exactly one vertex $\mathbf{v}_{0}$ for which $\mathbf{C}_{\mathbf{v}_{0}}^{\sharp}=\mathbf{C}_{\mathbf{v}_{0}}$ (namely the vertex $\mathbf{v}_{0}$ for which $\langle\xi, \mathbf{v}\rangle$ is minimal). Then, considering the outward dual (or normal cone) $\mathbf{N}_{\mathbf{v}_{0}}^{-}$to $\mathbf{C}_{\mathbf{v}_{0}}$ (i.e. the pointed cone generated by the outward normal vectors to the facets of $P$ that contain $\mathbf{v}_{0}$ ) and the set $\widetilde{W}$ defined in (18), one can take $\lambda>0$ such that $-\lambda \xi \in \mathbf{N}_{\mathbf{v}_{0}}^{-}$, while $\mathbf{v}_{0}-\lambda j(\xi) \in \widetilde{W}$ and

$$
\lambda>\max _{\substack{\text { vertex of } P \\ i=1, \ldots, L_{\mathbf{v}} \\ j=1, \ldots, d}}\left\{\frac{\left\langle j^{-1}\left(\mathbf{v}_{0}-\mathbf{v}\right), \alpha_{\mathbf{v}, i, j}\right\rangle}{\left\langle\xi, \alpha_{\mathbf{v}, i, j}\right\rangle}\right\},
$$

where the vectors $\alpha_{\mathbf{v}, i, j}$, for $i=1, \ldots, L_{\mathbf{v}}$ and $j=1, \ldots, d$, are the generators of the cones $\mathbf{C}_{\sigma_{i}(\mathbf{v})}$ given by a regular triangulation (the existence of $\lambda$ is guaranteed by the fact that $\xi$ is not perpendicular to any edge vector of the cones involved). Then, taking $\varepsilon:=\mathbf{v}_{0}-\lambda j(\xi)$ and $\beta_{\mathbf{v}}:=\mathbf{v}-\varepsilon$, one easily sees that

$$
\left\langle\xi, \alpha_{\mathbf{v}, i, j}\right\rangle\left\langle j^{-1}\left(\beta_{\mathbf{v}}\right), \alpha_{\mathbf{v}, i, j}\right\rangle=\left\langle\xi, \alpha_{\mathbf{v}, i, j}\right\rangle^{2} \lambda-\left\langle\xi, \alpha_{\mathbf{v}, i, j}\right\rangle\left\langle j^{-1}\left(\mathbf{v}_{0}-\mathbf{v}\right), \alpha_{\mathbf{v}, i, j}\right\rangle>0
$$

for every $i=1, \ldots, L_{\mathbf{v}}$ and $j=1, \ldots, d$, and so, given any vertex $\mathbf{v}$, polarizing the cones $\mathbf{C}_{\sigma_{i}(\mathbf{v})}$ with respect to the vector obtained from $\beta_{\mathbf{v}}$ is equivalent to polarizing them with respect to $\xi$.

Since Proposition 6.6 holds for any convex polytope, we conclude that, given any solid pointed non-simple cone $\mathbf{C}$ contained in a subspace $L \subset V$, 
any $\mathbf{x} \in L \backslash S_{\mathbf{v}}$ (where $S_{\mathbf{v}}$ is the union of the affine spaces of $L$ generated by non-simple faces of $\mathbf{C}$ ), and any assignment of weights to the hyperplanes generated by the facets of $\mathbf{C}$, the value of the weighted characteristic function $\mathbf{1}_{\mathcal{T}_{\mathbf{v}}}^{w}(\mathbf{x})$ of the polarized cone $\mathbf{C}^{\sharp}$ (with respect to some polarizing vector), obtained from a regular triangulation of the inner normal cone to the vertex $\mathbf{v}$ of $\mathbf{C}$, does not depend on the choice of the triangulation.

Going back to $V$ and taking any non-simple face $F$ of $P$, we note that for any $\mathbf{x} \in V \backslash S_{F}$,

$$
\mathbf{1}_{\mathcal{T}_{F}}^{w}(\mathbf{x})=\sum_{i=1}^{L_{F}}(-1)^{m_{F, i}} \mathbf{1}_{\mathbf{C}_{\sigma_{i}(F)}^{\sharp}}^{w}(\mathbf{x})=\sum_{i=1}^{L_{F}}(-1)^{m_{F, i}} \mathbf{1}_{\mathbf{T}_{\mathbf{C}_{\sigma_{i}(F)}^{\sharp}}^{w}}\left(\pi_{F}(\mathbf{x})\right),
$$

where $\mathbf{T}_{\mathbf{C}_{\sigma_{i}(F)}^{\sharp}}$ is the transverse cone of $\mathbf{C}_{\sigma_{i}(F)}^{\sharp}$ along $\Delta_{F}$; i.e., a fulldimensional pointed cone in $V / \operatorname{lin}(F)$ given by the image $\pi_{F}\left(\mathbf{C}_{\sigma_{i}(F)}^{\sharp}\right)$ of the affine cone $\mathbf{C}_{\sigma_{i}(F)}^{\sharp}$. Consequently, $\mathbf{1}_{\mathcal{T}_{F}}^{w}(\mathbf{x})$ is equal to the value of the characteristic function of the polarized transverse (pointed) cone along $F$ at the point $\pi_{F}(\mathbf{x})$, for some triangulation of $\mathbf{N}_{\pi_{F}\left(\Delta_{F}\right)}$ induced by a triangulation of $\mathbf{N}_{F}$ (note that we can naturally identify $\mathbf{N}_{\pi_{F}\left(\Delta_{F}\right)}$ to $\mathbf{N}_{F}$ ). Since we know that the values of this last function on $(V / \operatorname{lin}(F)) \backslash S_{\pi_{F}\left(\Delta_{F}\right)}$ are independent of the choice of a regular triangulation, so are the values of $\mathbf{1}_{\mathcal{T}_{F}}^{w}$ on $V \backslash S_{F}$, where $S_{F}$ is the union of the affine spaces generated by the non-simple faces of $P$ that contain $F$. Then, we obtain the following result.

Proposition 6.8. Let $F$ be a non-simple face of a convex polytope $P$. The values of the function $\mathbf{1}_{\mathcal{T}_{F}}^{w}$ on $V \backslash S_{F}$ are independent of the choice of a regular triangulation $\mathcal{T}_{F}$. Moreover, in the particular case where the weights $q_{i}$ are equal for all $i=1, \ldots, N_{P}$, the values of $\mathbf{1}_{\mathcal{T}_{F}}^{w}$ on the whole space $V$ do not depend on the choice of a regular triangulation.

After this, since we now know that these functions are independent of the regular triangulation chosen, we will denote them simply by $\mathbf{1}_{F}^{w}$ and finally obtain our main result (Theorem 1.1).

Remark 6.9. Choosing a regular triangulation of the dual polytope $P^{*}$ and obtaining, by restriction, regular triangulations of the dual cones $\mathbf{N}_{F}$ at non-simple faces $F$, corresponds to performing a simple deformation of the polytope obtained by parallel moving some of its facets. Hence, an alternative proof for the above decomposition is obtained by carefully choosing these small perturbations of $P$ and then applying Theorem 5.1. In particular, 
we proceed as follows. First, we express $P$ as an intersection of halfspaces. Then, for a fixed $\mathbf{x} \in V \backslash S$, we consider the halfspaces $H_{i}$ whose boundaries do not contain $\mathbf{x}$ and we shift them by sufficiently small amounts $h_{i}$ such that, if $\mathbf{x}$ is in the interior of $H_{i}$, it remains in its interior and, if it is in the exterior, it remains in the exterior. A generic choice of such shifts should yield a simple perturbation $P_{h}$ of $P$. Indeed, since $\mathbf{x}$ is not in any non-simple face of $P$, even if we restrict to this set of perturbations, the polytope $P_{h}$ will still be simple for an open dense subset of values of $h$ near zero. Then, the left and right hand sides of (16) in Proposition 6.2, when evaluated at $\mathbf{x}$, are the same for the simple perturbed polytope as they are for the original polytope $P$. However, this alternative argument for the proof of Proposition 6.2 cannot be applied if we want to extend our result to points in $S$, as we do in the particular case where all the weights $q_{i}$ are equal to some fixed value $q \in \mathbb{C}$. For this we would still need a wall-crossing argument. Note that this extended decomposition was used, in this particular case, in Propositions 6.6 and 6.8 to show that the values of the functions $\mathbf{1}_{F}^{w}$ on $V$ do not depend on the choice of a regular triangulation. In all cases, if we use the perturbation argument, the important fact that the functions $\mathbf{1}_{F}^{w}$ are independent of the choice of a regular triangulation (or of a simple perturbation) would still remain to be proved.

Finally, we point out that we can repeat the above arguments to show that the polytope decompositions presented in (12) (using $-\beta_{F}$ to obtain the polarizing vectors) also generalize to any convex polytope (we just multiply the functions $\mathbf{1}_{F}^{w}$ by $\left.(-1)^{\operatorname{dim} F}\right)$.

\section{Acknowledgments}

We are grateful to Yael Karshon for her corrections and suggestions on a previous version of this work. In particular, the alternative argument presented Remark 6.9, which uses simple perturbations of the polytope, is due to her.

Work done within the activities of Centro de Análise Funcional, Estruturas Lineares e Aplicações (Universidade de Lisboa) and Centro de Análise Matemática Geometria e Sistemas Dinâmicos CAMGSD (Instituto Superior Técnico).

JA was supported by Fundação para a Ciência e Tecnologia (FCT/Portugal) through the program Ciência 2008 and grant SFRH/BPD/111317/2015. 
The work of LG is partially supported by Fundação para a Ciência e Tecnologia (FCT/Portugal), projects EXCL/MAT-GEO/0222/2012, POCTI/ MAT/117762/2010 and UID/MAT/04459/2013.

\section{References}

[1] J. Agapito, A weighted version of quantization commutes with reduction for a toric manifold, Contemp. Math. 374 (2005), 1-14.

[2] J. Agapito, Weighted Brianchon-Gram decomposition, Canad. Math. Bull. 49 (2006), 161-169.

[3] J. Agapito and L. Godinho, New polytope decompositions and EulerMaclaurin formulas for simple integral polytopes, Adv. Math. 214 (2007), 379-416.

[4] J. Agapito and J. Weitsman, The weighted Euler-Maclaurin formula for a simple integral polytope, Asian J. Math. 9 (2005) 199-211.

[5] A. Barvinok, A course in convexity, Graduate Studies in Mathematics 54, AMS, RI. (2002).

[6] F. Battaglia, Geometric spaces from arbitrary convex polytopes, Internat. J. Math. 23 (2012), 1250013, 39 pp.

[7] C. Brianchon, Théorème nouveau sur les polyèdres, J. École polytechnique 15 (1837), 317-319.

[8] D. Burns, V. Guillemin, and E. Lerman, Toric symplectic singular spaces I. Isolated singularities, Conference on Symplectic Topology, J. Symplectic Geom. 3 (2005), 531-543.

[9] C. Haase, Polar decomposition and Brion's theorem, Contemp. Math. 374 (2005), 91-99.

[10] M. Harada and Y. Karshon, Localization for equivariant cohomology with varying polarization, Comm. Annal. Geom. 20 (2012), 869-947.

[11] K. Hori, S. Katz, A. Klemm, R. Pandharipande, R. Thomas, C. Vafa, R. Vakil, and E. Zaslow, Mirror symmetry, Clay Mathematics Monographs, 1, AMS, Providence, RI; Clay Mathematics Institute, Cambridge, MA, 2003.

[12] J.-M. Kantor and A. Khovanskii, Une application du théorème de Riemann-Roch combinatoire au polynôme d'Ehrhart des polytopes entiers de $\mathbb{R}^{d}$, C. R. Acad. Sci. Paris Sér. I Math. 317 (1993), 501-507. 
[13] Y. Karshon, S. Sternberg, and J. Weitsman, Euler-Maclaurin with remainder for a simple integral polytope, Duke Math. J. 130 (2005), 401434.

[14] J. Lawrence, Polytope volume computation, Math. Comp. 57 (1991), 259-271.

[15] C. W. Lee, Subdivisions and triangulations of polytopes, Handbook of discrete and computational geometry, 271-290, CRC Press Ser. Discrete Math. Appl., Boca Raton, 1997.

[16] P-E. Paradan, Formules de localisation en cohomologie equivariante, Compositio. Math. 117 (1999), 243-293.

[17] A. Varchenko, Combinatorics and topology of the arrangement of affine hyperplanes in the real space (Russian) Funktsional. Anal. i Prilozhen. 21, 11-22. English translation: Functional Anal. Appl. 21 (1987), 9-19.

Centro de Análise Funcional, Estruturas lineares e Aplicações Departamento de Matemática, Fac. Ciências Univ. de Lisboa 1749-016 Lisbon, Portugal

E-mail address: jaruiz@ciencias.ulisboa.pt

Centro de Análise Matemática, Geometria e Sistemas Dinâmicos Departamento de Matemática, Instituto Superior Técnico

Av. Rovisco Pais, 1049-001 Lisbon, Portugal

E-mail address: lgodin@math.tecnico.ulisboa.pt

ReCEIVEd FEBRUARY 25, 2014 